TRANSACTIONS OF THE

AMERICAN MATHEMATICAL SOCIETY

Volume 359, Number 9, September 2007, Pages 4557-4593

S 0002-9947(07)04262-6

Article electronically published on April 17, 2007

\title{
ALLEE EFFECT AND BISTABILITY IN A SPATIALLY HETEROGENEOUS PREDATOR-PREY MODEL
}

\author{
YIHONG DU AND JUNPING SHI
}

\begin{abstract}
A spatially heterogeneous reaction-diffusion system modelling predator-prey interaction is studied, where the interaction is governed by a Holling type II functional response. Existence of multiple positive steady states and global bifurcation branches are examined as well as related dynamical behavior. It is found that while the predator population is not far from a constant level, the prey population could be extinguished, persist or blow up depending on the initial population distributions, the various parameters in the system, and the heterogeneous environment. In particular, our results show that when the prey growth is strong, the spatial heterogeneity of the environment can play a dominant role for the presence of the Allee effect. Our mathematical analysis relies on bifurcation theory, topological methods, various comparison principles and elliptic estimates. We combine these methods with monotonicity arguments to the system through the use of some new auxiliary scalar equations, though the system itself does not keep an order structure as the competition system does. Among other things, this allows us to obtain partial descriptions of the dynamical behavior of the system.
\end{abstract}

\section{INTRODUCTION}

A system of differential equations has been used to model the predator-prey interaction between two populations since the pioneering work of Volterra and Lotka in the 1920s. When the spatial distributions of the two populations are also of interest, the passive dispersal of the populations can be modelled by a diffusion operator. Combining the growth, interaction and dispersal of the predator and prey, a natural mathematical model for their spatiotemporal behavior is a reactiondiffusion system of the form

$$
\left\{\begin{array}{l}
u_{t}-d_{1} \Delta u=\lambda u-a u^{2}-b \phi(u) v \\
v_{t}-d_{2} \Delta v=\mu v-d v^{2}+c \phi(u) v .
\end{array}\right.
$$

Here we assume that both the predator and prey populations have a logistic growth rate. The function $\phi(u)$ represents the functional response of the predator. The

Received by the editors April 6, 2005 and, in revised form, February 10, 2006.

2000 Mathematics Subject Classification. Primary 35J55, 92D40; Secondary 35B30, 35B32, 35J65, 92D25.

Key words and phrases. Reaction-diffusion system, predator-prey model, spatial heterogeneity.

The first author was partially supported by the Australia Research Council.

The second author was partially supported by United States NSF grants DMS-0314736 and EF-0436318, College of William and Mary junior research leave, and a grant from Science Council of Heilongjiang Province, China. 
classical Lotka-Volterra model assumes that $\phi(u)=u$. If the handling time of each prey is also considered, then a more reasonable response function is the Holling type II response $\phi(u)=u /(1+m u)$ for some $m>0[\mathrm{H}]$.

In the spatial predator-prey model (1.1), the parameters are traditionally assumed to be constant. However, it is more realistic to assume that the growth rate, the crowding effect, and the predator-prey interaction rates are spatially dependent, due to the heterogeneity of the environment. Hence we consider a reaction-diffusion system

$$
\begin{cases}u_{t}-d_{1} \Delta u=\lambda(x) u-a(x) u^{2}-\frac{b(x) u v}{1+m(x) u}, & x \in \Omega, t>0, \\ v_{t}-d_{2} \Delta v=\mu(x) v-d(x) v^{2}+\frac{c(x) u v}{1+m(x) u}, & x \in \Omega, t>0, \\ \partial_{\nu} u=\partial_{\nu} v=0, & x \in \partial \Omega, t>0, \\ u(x, 0)=u_{0}(x) \geq 0, v(x, 0)=v_{0}(x) \geq 0, & x \in \Omega .\end{cases}
$$

Here $\Omega$ is a bounded domain in $\mathbf{R}^{n}$ with smooth boundary and $n \geq 1$, the parameter functions are assumed to be nonnegative and continuous in $\bar{\Omega}$ (except $\mu(x)$, which can take negative values). $\partial_{\nu} u=\nabla u(x) \cdot \nu(x)$ is the out-flux of $u$, and $\nu(x)$ is the outer unit normal vector of $\partial \Omega$ at $x$. Thus the zero-flux boundary condition used in the system indicates it is a closed ecosystem in the habitat $\Omega$.

In this article, we are interested in the set of steady state solutions of (1.2) and the associated asymptotic dynamical behavior. In particular we are interested in the impact of a Holling type II response, instead of the more traditional LotkaVolterra model, and the effect of the spatial heterogeneity. In our mathematical analysis, we will make use of an interesting observation; namely, some monotonicity arguments can be applied to this kind of predator-prey system through some auxiliary scalar equations (see (3.12), (3.29), (4.22) and (4.36)). This observation will play a crucial role in our analysis; among other things, it enables us to obtain some descriptions of the dynamical behavior of the system, which seem difficult to get by other techniques.

To focus our interest, we shall only consider a more special model (see (2.1) below) with all parameter functions in (1.2) being constant except $a(x)$, which represents the crowding effect on the prey population. The function $a(x)$ is assumed to be degenerate on a subregion $\Omega_{0}$ of $\Omega$, i.e., $a(x)=0$ for $x \in \Omega_{0}$, and $a(x)>0$ otherwise. Biologically $\Omega_{0}$ is a more favorable subregion for the prey where its growth can be unbounded. Then an interesting question is whether an introduction of a predator will control the otherwise possibly unbounded growth of the prey population. The answer to this question is not intuitively obvious because we assume the prey-consumption ability of the predator is limited, obeying a Holling type II response.

We will show that there is a threshold prey growth rate $\lambda_{*}$, and the dynamics of the system undergoes a drastic change when $\lambda$ crosses this value. We shall call $\lambda<\lambda_{*}$ the weak prey growth, and $\lambda>\lambda_{*}$ the strong prey growth. In the weak prey growth case, the degeneracy of $a(x)$ does not seem to have a significant effect on the asymptotic behavior, which is similar to the case that $a(x)$ is positive in $\bar{\Omega}$. In particular, when $\mu$ is large, there is no positive steady-state, and the semitrivial steady state $(0, \mu)$ is globally asymptotically stable. Thus the prey population cannot evade extinction while the predator population stabilizes at the level $\mu$. 
When $\mu$ is positive and small, an Allee effect is observed. If the initial population of prey $u_{0}$ is large enough, a coexistence state can be reached eventually, but if $u_{0}$ is small, then the prey will become extinct; on the other hand, regardless of its initial (nontrivial) state, the population of the predator will always be no less than $\mu$ in the long time. We notice that the prey population does not blow up, although there is a region where it can grow without bound. We are able to partially characterize the long time dynamical behavior and its bistable property in certain parameter ranges (see Theorem 3.8). Moreover, we will examine the effect of $m$ on the bistability of the system and show that when $m$ is large, the bistable range of $\mu$ always exists, but as $m$ decreases, the bistable range of $\mu$ may shrink to empty.

A drastic change occurs when the prey growth rate $\lambda$ increases across the threshold value $\lambda_{*}$. In the strong prey growth case, the system has a positive steady-state for all $\mu>\lambda / b$, and the Allee effect can be observed for all positive $m$ and almost all predator growth rates $\mu$. Therefore even when $m$ is small (thus the predator's consumption ability is strong) and $\mu$ is large (the predator also grows fast), the prey population can still be out of control. Indeed we will show that if $u_{0}$ is large enough, then the prey population goes to infinity in $\Omega_{0}$ when $t \rightarrow \infty$, and when $u_{0}$ is small, the prey will become extinct as $t \rightarrow \infty$. This suggests that in the strong prey growth case, the degeneracy of the crowding effect function $a(x)$ plays a dominant role for the presence of the Allee effect. This is in sharp contrast to the weak prey growth case mentioned in the last paragraph, where the rate of saturation, namely the value of $m$, determines whether or not the Allee effect can be observed. However, we should be cautious that in the strong prey growth case, though dominating, the degeneracy of $a(x)$ alone does not generate the Allee effect; the presence of saturation (i.e., $m>0$ ) is important, though the rate of saturation is not (for the Allee effect). Indeed when $m=0$ (no saturation, classical Lotka-Volterra case), the system possesses a positive steady-state for all $\mu<\lambda / b$, and it has no positive steady-state when $\mu \geq \lambda / b$ (the predator only state is globally stable). Such results for classical Lotka-Volterra equations were obtained in [DD] for the Dirichlet boundary value problem, but they carry over to the Neumann problem as well. A more comprehensive comparison of the dynamics of the classical Lotka-Volterra and that of Holling type II predations can be found in our recent survey [DS2].

It is arguable whether such degeneracy $(a(x)=0)$ is realistic. However, the phenomena in the last paragraph largely remain if we replace the degenerate function $a(x)$ by $a(x)+\varepsilon$ for a small positive constant $\varepsilon$. Now in $\Omega_{0}$, unbounded growth of the prey is impossible, but the prey will have a rather large carrying capacity. We will show that for any fixed $m>0$ and $\mu>0$, the Allee effect exists if $\varepsilon$ is small enough. Thus, in this situation, the Allee effect is again mainly caused by the strong spatial heterogeneity of the environment (namely, the "near degeneracy" of the prey crowding effect) though the presence of saturation in the functional response is also important. We would like to remark that $\mu>0$ is possible if the predator has other food sources apart from the prey $u$. In Figure 1, an indication of the behavior of the global bifurcation branches of the positive steady-states is shown, where drastic changes can be observed between the cases $\lambda\left\langle\lambda_{*}\right.$ and $\left.\lambda\right\rangle \lambda_{*}$. As will become clear later, the multiplicity of steady state solutions depends further on the parameters $m$ and $\mu$.

Diffusive predator-prey systems have received much attention from ecologists and mathematicians since the 1970s; see, for example, SJ, SL, dMR, Co, Da2, BB] for 


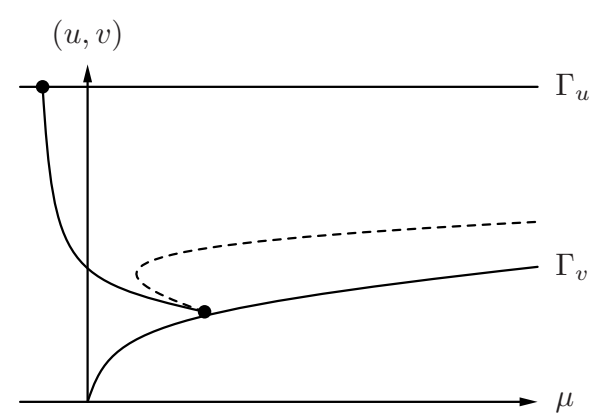

(a)

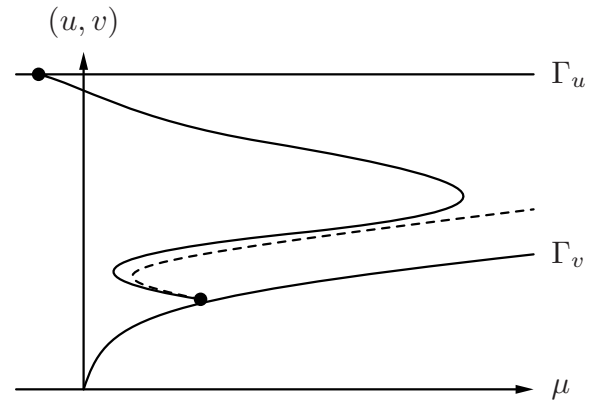

(b)

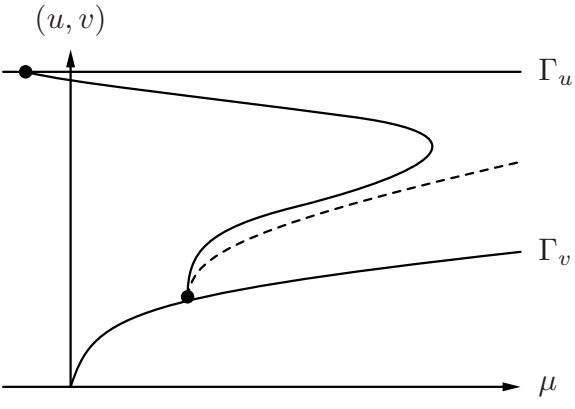

(c)

Figure 1. Possible bifurcation diagram of positive steady state solutions; solid line: $\lambda<\lambda_{*}$, dashed line: $\lambda>\lambda_{*}$.

some of the early research, where a homogeneous spatial environment was always assumed. Travelling wave solutions and pattern formation of predator-prey systems with type II predation but again in a spatially homogeneous environment have been studied in [HLR, MP, OL] (see also the references therein for related problems). Recently, the impact of degeneracy and heterogeneity of the environment has been studied in [D1, D2, D3] for the Lotka-Volterra competition model, and in [DD] for the Lotka-Volterra predator-prey model. More recently, a diffusive Holling-Tanner type predator-prey model was considered in $[\mathrm{DHs}$. In both $[\mathrm{DD}]$ and $\overline{\mathrm{DHs}}$, only one attracting state seems possible, contrasting the possible bistability for (1.2). On the other hand, when the environment is homogeneous $(a(x) \equiv a)$, the set of 
steady state solutions of (1.2) was studied in DL3] for the large $m$ case (see also BB, CEL, DL1, DL2] for Dirichlet problems), and for small $m$, it follows from results in dMR that the system has at most one positive steady-state, and it is a constant solution and is globally attracting (when it exists). In this paper, we consider a general $m>0$, and most of our results holds for all positive $m$. In particular we show that for intermediate $m$ (neither small nor large), (1.2) may have a global branch of steady state solutions whose shape is roughly a reversed $S$ (see Figure 1 (b)). In a related recent paper [DS1, we also consider the impact of a protect zone for the prey species on the dynamics. It is interesting that in that case, a unique globally stable positive steady-state exists for strong prey growth and strong predator growth although we still have a saturation on the predator functional, in contrast to the bistability we show in the current paper.

Allee effects and bistability in scalar reaction-diffusion equations were studied in KS, OS1, OS2, SS2 . In particular, the Allee effect appears in a scalar reactiondiffusion equation with logistic growth and Holling type II functional response predation. Compared to the results in [SS2], the dynamical behavior of (1.2) for weak prey growth is similar to the weak Allee effect in which bistability only exists for a bounded parameter range, and the behavior of (1.2) with strong prey growth is similar to the strong Allee effect where bistability exists for an unbounded parameter range. The bifurcation parameter in SS2 is the diffusion coefficient, and here we use the predator growth rate, but most of our results remain true if we use the diffusion coefficient as the bifurcation parameter (with suitable rescaling).

Our mathematical approach is based on bifurcation theory, topological methods, elliptic and parabolic comparison principles and various elliptic estimates. We will introduce the basic mathematical setup and recall some preliminary results in Section 2. We consider the weak and strong prey growth cases in Sections 3 and 4 respectively, and in Section 5, we consider the perturbed case when $a(x)$ is replaced by $a(x)+\varepsilon$. Though our predator-prey system has no monotonicity property (in contrast to the competition systems), we are able to make extensive use of some scalar equations (see (3.12), (3.29), (4.22) and (4.36)), where monotonicity arguments apply, to obtain various results on the global bifurcation branches of the steady-state solutions of our system and its dynamical behavior. Our main mathematical results are further explained in biological terms in subsections 3.4, 4.5 and 5.2 .

\section{BASIC SETUP AND PRELIMINARIES}

To concentrate on the impact of the degeneracy of $a(x)$ on the dynamics of (1.2), we assume that the other parameters in the equations are spatially homogeneous. Moreover, for the convenience of notation, we assume that the diffusion constants $d_{1}=d_{2}=1$. Thus (1.2) becomes

$$
\begin{cases}u_{t}-\Delta u=\lambda u-a(x) u^{2}-\frac{b u v}{1+m u}, & x \in \Omega, t>0, \\ v_{t}-\Delta v=\mu v-v^{2}+\frac{c u v}{1+m u}, & x \in \Omega, t>0, \\ \partial_{\nu} u=\partial_{\nu} v=0, & x \in \partial \Omega, t>0, \\ u(x, 0)=u_{0}(x) \geq 0, v(x, 0)=v_{0}(x) \geq 0, & x \in \Omega,\end{cases}
$$


and for the equation of steady state solutions, now we have

$$
\begin{cases}-\Delta u=\lambda u-a(x) u^{2}-\frac{b u v}{1+m u}, & x \in \Omega, \\ -\Delta v=\mu v-v^{2}+\frac{c u v}{1+m u}, & x \in \Omega, \\ \partial_{\nu} u=\partial_{\nu} v=0, & x \in \partial \Omega .\end{cases}
$$

Here $\lambda, \mu, b, c, m$ are constants, all positive except $\mu$, which may take negative values; $a(x)$ is a nonnegative continuous function on $\bar{\Omega}$. Moreover there exists a subregion $\Omega_{0}$ such that $\overline{\Omega_{0}} \subset \Omega$, and

$$
a(x) \equiv 0, \quad x \in \overline{\Omega_{0}}, \text { and } a(x)>0, x \in \bar{\Omega} \backslash \overline{\Omega_{0}} .
$$

We assume that $\Omega_{0}$ is an open and connected subset of $\Omega$, and it has $C^{2}$ boundary $\partial \Omega_{0}$.

Linear eigenvalue problems will play important roles in our analysis. We define $\lambda_{1}^{D}(\phi, O)$ and $\lambda_{1}^{N}(\phi, O)$ to be the principal eigenvalues of $-\Delta+\phi$ over the region $O$, with Dirichlet or Neumann boundary conditions respectively. If the region $O$ is omitted in the notation, then we understand that $O=\Omega$. If the potential function $\phi$ is omitted, then we understand that $\phi=0$. We recall some well-known properties of $\lambda_{1}^{D}(\phi, O)$ and $\lambda_{1}^{N}(\phi, O)$ :

(1) $\lambda_{1}^{D}(\phi, O)>\lambda_{1}^{N}(\phi, O)$;

(2) $\lambda_{1}^{B}\left(\phi_{1}, O\right)>\lambda_{1}^{B}\left(\phi_{2}, O\right)$ if $\phi_{1} \geq \phi_{2}$ and $\phi_{1} \not \equiv \phi_{2}$, for $B=D, N$;

(3) $\lambda_{1}^{D}\left(\phi, O_{1}\right) \geq \lambda_{1}^{D}\left(\phi, O_{2}\right)$ if $O_{1} \subset O_{2}$.

When describing the properties of solutions to (2.1), we will often need the knowledge of the scalar equation:

$$
\begin{cases}u_{t}-\Delta u=\lambda u-a(x) u^{2}, & x \in \Omega, t>0, \\ \partial_{\nu} u=0, & x \in \partial \Omega, t>0, \\ u(x, 0)=u_{0}(x) \geq 0, & x \in \Omega .\end{cases}
$$

The corresponding steady state problem is

$$
-\Delta u=\lambda u-a(x) u^{2}, x \in \Omega, \partial_{\nu} u=0, x \in \partial \Omega .
$$

It is well known (see $\left[\mathrm{O}\right.$ ) that the eigenvalue $\lambda_{1}^{D}\left(\Omega_{0}\right)=\lambda_{1}^{D}\left(0, \Omega_{0}\right)>0$ plays an important role in the dynamics of (2.4); it is also known that to fully describe the behavior of (2.4) one needs the boundary blow-up problem (see [DHu]):

$$
-\Delta u=\lambda u-a(x) u^{2}, x \in \Omega \backslash \overline{\Omega_{0}}, \quad \partial_{\nu} u=0, x \in \partial \Omega, u=\infty, x \in \partial \Omega_{0} .
$$

The main results on (2.6), (2.5) and (2.4) can be summarized as follows (see $[\mathrm{DHu}]$ ):

Theorem 2.1 (Boundary blow-up problem). (1) For any $\lambda \in \mathbf{R},(2.6)$ has a minimal positive solution $\underline{U}^{\lambda}$ and a maximal positive solution $\bar{U}^{\lambda}$.

(2) If there exist positive constants $\gamma$ and $\beta$ such that $\lim _{x \rightarrow \partial \Omega_{0}} a(x) /[d(x, \partial \Omega)]^{\gamma}$ $=\beta$, then (2.6) has a unique positive solution $U^{\lambda}$.

Theorem 2.2 (Steady state solutions). (1) (2.5) has a unique positive solution $u_{\lambda}(x)$ when $0<\lambda<\lambda_{1}^{D}\left(\Omega_{0}\right)$, and it has no positive solution when $\lambda \geq \lambda_{1}^{D}\left(\Omega_{0}\right)$.

(2) $u_{\lambda} \rightarrow 0$ uniformly in $\Omega$ as $\lambda \rightarrow 0^{+}$, and $u_{\lambda} \rightarrow \infty$ uniformly on $\overline{\Omega_{0}}$ as $\lambda \rightarrow\left[\lambda_{1}^{D}\left(\Omega_{0}\right)\right]^{-}$, and $u_{\lambda} \rightarrow \underline{U}^{\lambda}$ uniformly on any compact subset of $\bar{\Omega} \backslash \overline{\Omega_{0}}$ as $\lambda \rightarrow\left[\lambda_{1}^{D}\left(\Omega_{0}\right)\right]^{-}$. 
Theorem 2.3 (Dynamical behavior). ally asymptotically stable for (2.4).

(2) When $\lambda \geq \lambda_{1}^{D}\left(\Omega_{0}\right)$, $u(x, t) \rightarrow \infty$ for $x \in \overline{\Omega_{0}}$ as $t \rightarrow \infty$, and $\underline{U}^{\lambda}(x) \leq$ $\underline{\lim }_{t \rightarrow \infty} u(x, t) \leq \varlimsup_{t \rightarrow \infty} u(x, t) \leq \bar{U}^{\lambda}(x)$ for $x \in \bar{\Omega} \backslash \overline{\Omega_{0}}$.

Remark 2.4. The uniqueness result in Part 2 of Theorem 2.1 holds under weaker conditions; by [D6, the uniqueness holds if $\beta_{1} \leq a(x) /[d(x, \partial \Omega)]^{\gamma} \leq \beta_{2}$ for some $\beta_{1}, \beta_{2}, \gamma>0$ and all $x$ near $\partial \Omega$.

In the following analysis we will fix $\lambda$ for the two distinctive cases:

(a) weak prey growth: $0<\lambda<\lambda_{1}^{D}\left(\Omega_{0}\right)$, (b) strong prey growth: $\lambda>\lambda_{1}^{D}\left(\Omega_{0}\right)$,

and we will use the predator growth rate $\mu$ as a bifurcation parameter. We also fix the parameters $b, c, m>0$ unless otherwise stated. In the following, for simplicity of the notation, we will write $p(u)=u /(1+m u)$. It is easy to see that

$$
p(u)=\frac{u}{1+m u}, \quad p^{\prime}(u)=\frac{1}{(1+m u)^{2}}, \quad p^{\prime \prime}(u)=\frac{-2 m}{(1+m u)^{3}} .
$$

\section{WEAK PREY GROWTH RATE}

In this section we assume that $0<\lambda<\lambda_{1}^{D}\left(\Omega_{0}\right)$, and we will consider the structure of the set of nonnegative steady state solutions of (2.1) and its underlying dynamical behavior.

3.1. Steady state solutions: bifurcation analysis. For any $\mu>0,(2.2)$ has two semi-trivial solutions: $\left(u_{\lambda}, 0\right)$ and $(0, \mu)$. So we have two curves of these solutions in the space of $(\mu, u, v)$ :

$$
\Gamma_{u}=\left\{\left(\mu, u_{\lambda}, 0\right):-\infty<\mu<\infty\right\}, \quad \Gamma_{v}=\{(\mu, 0, \mu): 0<\mu<\infty\} .
$$

By the strong maximum principle, any nonnegative solution $(u, v)$ of (2.2) is either $(0,0)$, or a semi-trivial one, or a positive one.

From standard local bifurcation analysis, there is a bifurcation point $\mu_{1}=$ $\lambda_{1}^{N}\left(-c p\left(u_{\lambda}\right)\right)$ such that a smooth curve $\Gamma_{1}^{\prime}$ of positive solutions to (2.2) bifurcates from $\Gamma_{u}$ at $(\mu, u, v)=\left(\mu_{1}, u_{\lambda}, 0\right)$. Similarly, there is another bifurcation point $\mu_{2}=\lambda / b$ such that a smooth curve of positive solutions $\Gamma_{2}^{\prime}$ to (2.2) bifurcates from $\Gamma_{v}$ at $(\mu, u, v)=\left(\mu_{2}, 0, \mu_{2}\right)$. From global bifurcation theory, each of $\Gamma_{1}^{\prime}$ and $\Gamma_{2}^{\prime}$ is contained in a global branch of positive solutions to (2.2). We call these branches $\Gamma_{1}\left(\supset \Gamma_{1}^{\prime}\right)$ and $\Gamma_{2}\left(\supset \Gamma_{2}^{\prime}\right)$ respectively. Then, as in $\mathrm{BB}$ either $\Gamma_{1}$ and $\Gamma_{2}$ are both unbounded, or $\Gamma_{1}=\Gamma_{2}$. To show that the latter is the case, we prove the following lemma:

Lemma 3.1. Suppose that $0<\lambda<\lambda_{1}^{D}\left(\Omega_{0}\right)$ is fixed, and $(u, v)$ is a positive solution of (2.2). Then the following hold:

(1) $0<u(x)<u_{\lambda}(x)$, and $\mu+c / m>v(x)>\max \{\mu, 0\}$, where $u_{\lambda}$ is the unique positive solution of (2.5).

(2) The parameter $\mu$ must satisfy

$$
-\frac{c}{m}<\lambda_{1}^{N}\left(-c p\left(u_{\lambda}\right)\right)<\mu<\frac{\lambda\left(1+m\left\|u_{\lambda}\right\|_{\infty}\right)}{b} .
$$


Proof. Part 1 is a simple consequence of a standard comparison argument. For Part 2, from Part 1 and the equations, we have

$$
\lambda=\lambda_{1}^{N}\left(a(x) u+\frac{b v}{1+m u}\right)>\lambda_{1}^{N}\left(\frac{b \max \{\mu, 0\}}{1+m\left\|u_{\lambda}\right\|_{\infty}}\right)=\frac{b \max \{\mu, 0\}}{1+m\left\|u_{\lambda}\right\|_{\infty}},
$$

and since $p(u)$ is increasing,

$$
\mu=\lambda_{1}^{N}\left(v-\frac{c u}{1+m u}\right)>\lambda_{1}^{N}\left(-\frac{c u_{\lambda}}{1+m u_{\lambda}}\right)>\lambda_{1}^{N}\left(-\frac{c}{m}\right)=-\frac{c}{m} .
$$

From Lemma 3.1 we conclude that (2.2) has no unbounded branches of positive solutions when $0<\lambda<\lambda_{1}^{D}\left(\Omega_{0}\right)$ is fixed. Thus the two local branches must be connected. To obtain a more precise local picture of $\Gamma \equiv \Gamma_{1}=\Gamma_{2}$, we give more details on the bifurcation analysis. We apply the bifurcation result of CrandallRabinowitz [CR1, and we use a change of variables $v=\mu+w$ (so that $(u, w)=(0,0)$ is the trivial solution). For $p>1$, let $X=\left\{u \in W^{2, p}(\Omega): \partial_{\nu} u=0\right\}$, and let $Y=L^{p}(\Omega)$. Define $F: \mathbf{R} \times X \times X \rightarrow Y \times Y$ by

$$
F(\mu, u, w)=\left(\begin{array}{c}
\Delta u+\lambda u-a(x) u^{2}-b p(u)(\mu+w) \\
\Delta w-\mu w-w^{2}+c p(u)(\mu+w)
\end{array}\right) .
$$

We consider the bifurcation at $(\mu, u, w)=(\lambda / b, 0,0)$. From calculations,

$$
\begin{aligned}
& F_{(u, w)}(\mu, u, w)[\phi, \psi]=\left(\begin{array}{c}
\Delta \phi+\lambda \phi-2 a(x) u \phi-b p^{\prime}(u)(\mu+w) \phi-b p(u) \psi \\
\Delta \psi-\mu \psi-2 w \psi+c p^{\prime}(u)(\mu+w) \phi+c p(u) \psi
\end{array}\right), \\
& F_{\mu}(\mu, u, w)=\left(\begin{array}{c}
-b p(u) \\
-w+c p(u)
\end{array}\right), F_{\mu(u, w)}(\mu, u, w)[\phi, \psi]=\left(\begin{array}{c}
-b p^{\prime}(u) \phi \\
-\psi+c p^{\prime}(u) \phi
\end{array}\right), \\
& F_{(u, w)(u, w)}(\mu, u, w)[\phi, \psi]^{2}=\left(\begin{array}{c}
-2 a(x) \phi^{2}-2 b p^{\prime}(u) \phi \psi-b p^{\prime \prime}(u)(\mu+w) \phi^{2} \\
-2 \psi^{2}+2 c p^{\prime}(u) \phi \psi+c p^{\prime \prime}(u)(\mu+w) \phi^{2}
\end{array}\right) .
\end{aligned}
$$

At $(\mu, u, w)=(\lambda / b, 0,0)$, it is easy to verify that the kernel $\mathcal{N}\left(F_{(u, w)}(\lambda / b, 0,0)\right)=$ $\operatorname{span}\{(1, c)\}$, the range $\mathcal{R}\left(F_{(u, w)}(\lambda / b, 0,0)\right)=\left\{(f, g) \in Y^{2}: \int_{\Omega} f(x) d x=0\right\}$, and $F_{\mu(u, w)}(\lambda / b, 0,0)[1, c]=[-b, 0] \notin \mathcal{R}\left(F_{(u, w)}(\lambda / b, 0,0)\right)$. Thus we can apply the result of [CR1] to conclude that the set of positive solutions to (2.2) near $(\lambda / b, 0, \mu)$ is a smooth curve

$$
\Gamma_{2}=\left\{\left(\mu_{2}(s), u_{2}(s), \mu+w_{2}(s)\right): s \in(0, \delta)\right\},
$$

such that $\mu_{2}(0)=\lambda / b, u_{2}(s)=s+o(|s|), w_{2}(s)=c s+o(|s|)$. Moreover $\mu_{2}^{\prime}(0)$ can be calculated (see for example [S1]):

$$
\begin{aligned}
\mu_{2}^{\prime}(0) & =-\frac{\left\langle F_{(u, w)(u, w)}(\lambda / b, 0,0)[1, c]^{2}, l_{2}\right\rangle}{2\left\langle F_{\mu(u, w)}(\lambda / b, 0,0)[1, c], l_{2}\right\rangle} \\
& =-\frac{\int_{\Omega} a(x) d x}{b|\Omega|}-c+m \mu=\frac{-\bar{a}-b c+m \lambda}{b},
\end{aligned}
$$

where $\bar{a}=|\Omega|^{-1} \int_{\Omega} a(x) d x$, and $l_{2}$ is a linear functional on $Y^{2}$ defined as $\left\langle[f, g], l_{2}\right\rangle=$ $\int_{\Omega} f(x) d x$.

We can do a similar analysis at $\left(\mu_{1}, u_{\lambda}, 0\right)$, where $\mu_{1}=\lambda_{1}^{N}\left(-c p\left(u_{\lambda}\right)\right)$. We change the variables $w=u_{\lambda}-u$. Define

$$
G(\mu, u, v)=\left(\begin{array}{c}
\Delta w+\lambda w-2 a u_{\lambda} w+a w^{2}+b p\left(u_{\lambda}-w\right) v \\
\Delta v+\mu v-v^{2}+c p\left(u_{\lambda}-w\right) v
\end{array}\right) .
$$


Then

$$
\begin{array}{r}
G_{(w, v)}(\mu, w, v)[\phi, \psi] \\
=\left(\begin{array}{c}
\Delta \phi+\lambda \phi-2 a u_{\lambda} \phi+2 a w \phi-b p^{\prime}\left(u_{\lambda}-w\right) v \phi+b p\left(u_{\lambda}-w\right) \psi \\
\Delta \psi+\mu \psi-2 v \psi-c p^{\prime}\left(u_{\lambda}-w\right) v \phi+c p\left(u_{\lambda}-w\right) \psi
\end{array}\right), \\
G_{\mu}(\mu, w, v)=\left(\begin{array}{c}
0 \\
v
\end{array}\right), G_{\mu(w, v)}(\mu, u, w)[\phi, \psi]=\left(\begin{array}{c}
0 \\
\psi
\end{array}\right) \\
G_{(w, v)(w, v)}(\mu, w, v)[\phi, \psi]^{2}=\left(\begin{array}{c}
2 a \phi^{2}-2 b p^{\prime}\left(u_{\lambda}-w\right) \phi \psi+b p^{\prime \prime}\left(u_{\lambda}-w\right) v \phi^{2} \\
-2 \psi^{2}-2 c p^{\prime}\left(u_{\lambda}-w\right) \phi \psi+c p^{\prime \prime}\left(u_{\lambda}-w\right) v \psi^{2}
\end{array}\right) .
\end{array}
$$

At $(\mu, u, w)=\left(\mu_{1}, 0,0\right)$, it is easy to verify that the kernel $\mathcal{N}\left(G_{(w, v)}\left(\mu_{1}, 0,0\right)\right)=$ $\operatorname{span}\left\{\left(\varphi_{1}, \varphi_{2}\right)\right\}$, where $\left(\varphi_{1}, \varphi_{2}\right)$ satisfies

$$
\begin{cases}\Delta \phi+\lambda \phi-2 a(x) u_{\lambda} \phi+b p\left(u_{\lambda}\right) \psi=0, & x \in \Omega, \\ \Delta \psi+\mu \psi+c p\left(u_{\lambda}\right) \psi=0, & x \in \Omega, \\ \partial_{\nu} \phi=\partial_{\nu} \psi=0, & x \in \partial \Omega .\end{cases}
$$

Since $\mu=\mu_{1}^{N}\left(-c p\left(u_{\lambda}\right)\right)$, then we can choose $\varphi_{2}>0$. On the other hand, $u_{\lambda}$ is a stable solution of (2.5); thus the inverse of $L\left(u_{\lambda}\right)=\Delta+\lambda-2 a(x) u_{\lambda}$ is a negative operator, and $\varphi_{1}=\left[L\left(u_{\lambda}\right)\right]^{-1}\left(-b p\left(u_{\lambda}\right) \varphi_{2}\right)>0$. The range $\mathcal{R}\left(G_{(w, v)}\left(\mu_{1}, 0,0\right)\right)=$ $\left\{(f, g) \in Y^{2}: \int_{\Omega} g(x) \varphi_{2}(x) d x=0\right\}$, and $G_{\mu(w, v)}\left(\mu_{1}, 0,0\right)\left[\varphi_{1}, \varphi_{2}\right]=\left[0, \varphi_{2}\right] \notin$ $\mathcal{R}\left(G_{(w, v)}\left(\mu_{1}, 0,0\right)\right)$ since $\int_{\Omega} \varphi_{2}^{2} d x>0$. Thus we can apply the result of [CR1] to conclude that the set of positive solutions to (2.2) near $\left(\mu_{1}, u_{\lambda}, 0\right)$ is a smooth curve

$$
\Gamma_{1}=\left\{\left(\mu_{1}(s), u_{\lambda}(x)-u_{1}(s), v_{1}(s)\right): s \in[0, \delta)\right\},
$$

with $\mu_{1}(0)=\mu_{1}^{N}\left(-c p\left(u_{\lambda}\right)\right), u_{1}(s)=s \varphi_{1}(x)+o(|s|), w(s)=s \varphi_{2}(x)+o(|s|)$. Moreover,

$$
\begin{aligned}
\mu_{1}^{\prime}(0) & =-\frac{\left\langle G_{(w, v)(w, v)}\left(\mu_{1}, 0,0\right)\left[\varphi_{1}, \varphi_{2}\right]^{2}, l_{1}\right\rangle}{2\left\langle G_{\mu(w, v)}\left(\mu_{1}, 0,0\right)\left[\varphi_{1}, \varphi_{2}\right], l_{1}\right\rangle} \\
& =\frac{\int_{\Omega} \varphi_{2}^{3}(x) d x+c \int_{\Omega}\left(1+m u_{\lambda}(x)\right)^{-2} \varphi_{2}^{2}(x) \varphi_{1}(x) d x}{\int_{\Omega} \varphi_{2}^{2}(x) d x}>0,
\end{aligned}
$$

where $l_{1}$ is a linear functional on $Y^{2}$ defined as $\left\langle[f, g], l_{1}\right\rangle=\int_{\Omega} g(x) \varphi_{2}(x) d x$.

We summarize the above results on global bifurcation in the following theorem:

Theorem 3.2. Suppose that $0<\lambda<\lambda_{1}^{D}\left(\Omega_{0}\right)$ is fixed. Then there exists a continuum $\Gamma$ of positive solutions of (2.2) satisfying

$$
\operatorname{proj}_{\mu} \Gamma=\left(\bar{\mu}_{*}, \bar{\mu}^{*}\right] \text { or }\left(\bar{\mu}_{*}, \bar{\mu}^{*}\right),
$$

where

$$
\bar{\mu}_{*}=\lambda_{1}^{N}\left(-c p\left(u_{\lambda}\right)\right), \quad \lambda / b \leq \bar{\mu}^{*} \leq \lambda b^{-1}\left(1+m\left\|u_{\lambda}\right\|_{\infty}\right),
$$

and $\Gamma$ contains $\Gamma_{1}$ (defined in (3.8)) and $\Gamma_{2}$ (defined in (3.4)). Moreover, the bifurcation of $\Gamma_{1}$ at $\left(\mu_{1}^{N}\left(-c p\left(u_{\lambda}\right)\right), u_{\lambda}, 0\right)$ is supercritical $\left(\mu_{1}^{\prime}(0)>0\right)$, and the bifurcation of $\Gamma_{2}$ at $(\lambda / b, 0, \lambda / b)$ is supercritical $\left(\mu_{2}^{\prime}(0)>0\right)$ if $m>m_{0}$ and it is subcritical $\left(\mu_{2}^{\prime}(0)<0\right)$ if $0 \leq m<m_{0}$, where $m_{0}$ is defined as

$$
m_{0}=\lambda^{-1}[\bar{a}+b c], \quad \text { where } \bar{a}=\frac{1}{|\Omega|} \int_{\Omega} a(x) d x .
$$


3.2. Steady state solutions: multiplicity. In this subsection, we investigate in more detail the global bifurcation branch $\Gamma$ obtained above. It is well known that a predator-prey system does not possess an order structure to which a standard monotone method can be applied. However we will study an auxiliary scalar equation which has an order structure, and apply the results on the scalar equation to the original predator-prey system. Consider

$$
-\Delta u=\lambda u-a(x) u^{2}-b \mu \frac{u}{1+m u}, x \in \Omega, \partial_{\nu} u=0, x \in \partial \Omega .
$$

Proposition 3.3. Suppose that $0<\lambda<\lambda_{1}^{D}\left(\Omega_{0}\right)$ and that $b, m>0$ are fixed. Then $\mu=\lambda / b$ is a bifurcation point for (3.12) such that a global unbounded continuum $\Sigma$ of positive solutions of (3.12) emanates from $(\mu, u)=(\lambda / b, 0)$, and

$$
\operatorname{proj}_{\mu} \Sigma=\left(-\infty, \hat{\mu}^{*}\right] \text { or }\left(-\infty, \hat{\mu}^{*}\right) \text {, }
$$

where $\hat{\mu}^{*}=\sup \{\mu>0:$ (3.12) has a positive solution $\} \geq \lambda /$ b. Moreover $\Sigma$ satisfies the following:

(1) $\operatorname{Near}(\mu, u)=(\lambda / b, 0), \Sigma$ is a curve.

(2) When $\mu \leq 0$, (3.12) has a unique positive solution $\bar{U}_{\mu}(x)$, and $\left\{\left(\mu, \bar{U}_{\mu}\right)\right.$ : $\mu \leq 0\}$ is a smooth curve.

(3) For $\mu \in\left(-\infty, \hat{\mu}^{*}\right)$, (3.12) has a maximal positive solution $\bar{U}_{\mu}(x)$, and $\bar{U}_{\mu}$ is strictly decreasing with respect to $\mu$.

(4) For $\mu \in(-\infty, \lambda / b)$, (3.12) has a minimal positive solution $\underline{U}_{\mu}(x)$, and $\underline{U}_{\mu}(x) \equiv \bar{U}_{\mu}(x)$ when $\mu \leq 0$, and $\underline{U}_{\mu}$ is strictly decreasing with respect to $\mu$.

(5) If $\hat{\mu}^{*}>\lambda / b$, then (3.12) has a maximal positive solution for $\mu=\hat{\mu}^{*}$ and has at least two positive solutions for $\mu \in\left(\lambda / b, \hat{\mu}^{*}\right)$.

(6) If $\hat{\mu}^{*}>\lambda / b$ and $0<m<m_{0}$, then there exists $\hat{\mu}_{*} \in(0, \lambda / b)$ such that (3.12) has at least three positive solutions for $\mu \in\left(\hat{\mu}_{*}, \lambda / b\right)$ and $\underline{U}_{\mu}(x)<$

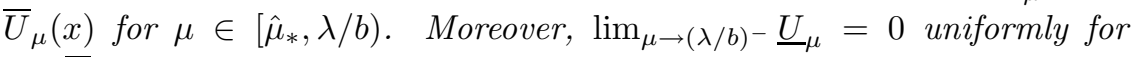
$x \in \bar{\Omega}$.

All these solutions mentioned above can be chosen from the unbounded continuum $\Sigma$.

Proof. The local bifurcation analysis is similar to the one in subsection 3.1. For $\mu \leq 0, f(x, u)=\lambda u-a(x) u^{2}-b \mu u /(1+m u)$ is a concave function in $U$ such that $f(x, 0)=0$ and $f_{u}(x, 0)>0$; thus (3.12) is a logistic type equation. Existence and uniqueness of a positive solution is well known in that case (see [CC1, CC2, SS2]). It is also easy to see that the unique solution $\bar{U}_{\mu}$ is globally asymptotically stable for the corresponding parabolic equation when $\mu \leq 0$. In particular, $\bar{U}_{\mu}$ is nondegenerate, so $\left\{\left(\mu, \bar{U}_{\mu}\right): \mu \leq 0\right\}$ is a smooth curve. Note that when $\mu=0$, $\bar{U}_{\mu} \equiv u_{\lambda}$, the unique positive solution of (2.5). On the other hand, as in the proof of Lemma 3.1] if $(\mu, u)$ is a solution of (3.12), then $\mu<\lambda b^{-1}\left(1+m\left\|u_{\lambda}\right\|_{\infty}\right)$. Hence the projection of $\Sigma$ on the $\mu$-axis is bounded from above, and we denote the upper bound by $\hat{\mu}^{*}$.

Define

$$
\tilde{\mu}^{*}=\sup \{\mu>0: \text { (3.12) has a positive solution }\} \text {. }
$$

We will later make use of a monotonicity argument to show that $\tilde{\mu}^{*}=\hat{\mu}^{*}$. If $0<\mu<\widetilde{\mu}^{*}$, for any positive solution $\widetilde{u}_{\mu}$ of (3.12), we have $\widetilde{u}_{\mu}<u_{\lambda}$ from a 
well-known lemma for logistic type equations (see, for example, [DM, Lemma 2.1] or [SY, Lemma 2.3]). So if we use $\widetilde{u}_{\mu}$ as a subsolution, $u_{\lambda}$ as a supersolution, and take the iteration sequence in a standard monotone method with $u_{\lambda}$ as the initial point, then the limit of the iteration is a positive solution $\bar{U}_{\mu}$ of (3.12), and $\bar{U}_{\mu} \geq \widetilde{u}_{\mu}$. Since $\widetilde{u}_{\mu}$ can be chosen as any positive solution of (3.12), $\bar{U}_{\mu}$ is the maximal positive solution of (3.12). The monotonicity of $\bar{U}_{\mu}$ in $\mu$ can be shown by using the maximality of $\bar{U}_{\mu}$ and the sub-supersolution method (for details see, for example, [SS1]).

We shall show that if $\widetilde{\mu}^{*}>\lambda / b$, then (3.12) still has a positive solution at $\mu=\widetilde{\mu}^{*}$. Indeed from the decreasing property of $\bar{U}_{\mu}, \bar{U}_{\widetilde{\mu}^{*}}=\lim _{\mu \rightarrow\left(\widetilde{\mu}^{*}\right)^{-}} \bar{U}_{\mu}$ exists and $\bar{U}_{\widetilde{\mu}^{*}} \geq 0$ is a solution of (3.12). $U_{\widetilde{\mu}^{*}} \not \equiv 0$ since $\widetilde{\mu}^{*}>\lambda / b$, and $\mu=\lambda / b$ is the only bifurcation point for positive solutions. Thus $\bar{U}_{\widetilde{\mu}^{*}}>0$ from the strong maximum principle.

Next we prove that all the maximal solutions $\bar{U}_{\mu}$ must be on the unbounded branch $\Sigma$, and thus $\widetilde{\mu}^{*}=\hat{\mu}^{*}$. Since $\bar{U}_{\mu}$ is the unique positive solution and it is nondegenerate when $\mu \leq 0$, we find that $\left\{\left(\mu, \bar{U}_{\mu}\right):-\infty<\mu \leq \delta\right\}$ for some $\delta>0$ is a smooth curve contained in $\Sigma$. For any $\mu_{0} \in\left(\delta, \widetilde{\mu}^{*}\right)$, define

$$
I_{\mu_{0}}=\left\{u \in C(\bar{\Omega}): \bar{U}_{0}(x) \geq u(x) \geq \bar{U}_{\mu_{0}}(x)\right\} .
$$

Then the projection of $\left\{\left(\mu, \bar{U}_{\mu}\right): 0<\mu \leq \delta\right\}$ onto $C(\bar{\Omega})$ is in $I_{\mu_{0}}$ from the monotonicity of $\bar{U}_{\mu}$. On the other hand, since $\Sigma^{\delta}:=\Sigma \backslash\left\{\left(\mu, \bar{U}_{\mu}\right): \mu \leq \delta\right\}$ is connected, and its closure contains $(\lambda / b, 0) \notin\left[0, \mu_{0}\right] \times I_{\mu_{0}}$, we must have $\Sigma^{\delta} \cap \partial\left(\left[0, \mu_{0}\right] \times I_{\mu_{0}}\right) \neq \emptyset$. Let $\left(\mu_{*}, u_{*}\right) \in \Sigma^{\delta} \cap \partial\left(\left[0, \mu_{0}\right] \times I_{\mu_{0}}\right)$; then $\left(\mu_{*}, u_{*}\right)$ is a solution of (3.12). First, $\mu_{*} \leq \delta$ is impossible since for such $\mu^{*}$, we necessarily have $u_{*}=\bar{U}_{\mu_{*}}$.

To consider the possibility of $\mu_{*} \in\left(\delta, \mu_{0}\right)$, we define an operator

$$
B(\mu, u)=(-\Delta+K)^{-1}\left((K+\lambda) u-a(x) u^{2}-b \mu u /(1+m u)\right)
$$

for $\mu \in \mathbf{R}$ and $u \in I_{\mu_{0}}$, where $K>0$ is a constant such that $f_{1}(x, u)=(K+\lambda) u-$ $a(x) u^{2}-b \mu u /(1+m u)$ is a positive increasing function for all $0 \leq u \leq \max \bar{U}_{0}(x)$ and $0 \leq \mu \leq \mu_{0}$. Thus $B(\mu, u)$ is a compact positive operator on $[0, \mu] \times I_{\mu}, B(\mu, u)$ is increasing with respect to $u$, and $B(\mu, u)=u$ if and only if $(\mu, u)$ is a solution of (3.12). Now if $\mu_{*} \in\left(\delta, \mu_{0}\right)$, then $u_{*} \geq \bar{U}_{\mu_{0}}$, and

$$
u_{*}=B\left(\mu_{*}, u_{*}\right)>B\left(\mu_{0}, u_{*}\right) \geq B\left(\mu_{0}, \bar{U}_{\mu_{0}}\right)=\bar{U}_{\mu_{0}} .
$$

Similarly, $u_{*}<\bar{U}_{0}$. Thus $u_{*}$ is in the interior of $I_{\mu_{0}}$, which contradicts with the definition of $\left(\mu_{*}, u_{*}\right)$. Therefore $\mu_{*}=\mu_{0}$, and $u_{*} \geq \bar{U}_{\mu_{0}}$. But $\bar{U}_{\mu_{0}}$ is maximal, and hence $u_{*}=\bar{U}_{\mu_{0}}$. In particular $\left(\mu_{0}, \bar{U}_{\mu_{0}}\right) \in \Sigma^{\delta}$ for any $\mu_{0} \in\left(\delta, \widetilde{\mu}^{*}\right)$. Therefore $\widetilde{\mu}^{*}=\hat{\mu}^{*}$. This finishes the proof of Parts 1-3.

For each fixed $\mu \in(-\infty, \lambda / b), u(x)=k$ is a subsolution of (3.12) for all small constant $k>0$, and we can assume $\bar{U}_{\mu}>k$. So if we use $\bar{U}_{\mu}$ as a supersolution, and $k$ as a subsolution, we can find a minimal solution $\underline{U}_{\mu}^{k}$ such that $k<\underline{U}_{\mu}^{k} \leq \bar{U}_{\mu}$. Clearly $\underline{U}_{\mu}^{k}$ is nonincreasing with respect to $k$, and $\underline{U}_{\mu}:=\lim _{k \rightarrow 0} \underline{U}_{\mu}^{k}$ is a nonnegative solution of (3.12). Since 0 is an isolated nonnegative solution, $\underline{U}_{\mu}$ must be a positive solution, and by its definition, it is the minimal positive solution.

Now we assume $\hat{\mu}^{*}>\lambda / b$ and prove Parts 5 and 6 . Note that by the argument before the last paragraph, we have that $\Sigma^{\delta} \cap \partial\left(\left[0, \hat{\mu}^{*}\right] \times I_{\hat{\mu}^{*}}\right)=\left\{\left(\hat{\mu}^{*}, \bar{U}_{\hat{\mu}^{*}}\right)\right\}$. Moreover from the saddle-node bifurcation theorem of Crandall and Rabinowitz [CR2, near $\left(\hat{\mu}^{*}, \bar{U}_{\hat{\mu}^{*}}\right), \Sigma^{\delta}$ is a curve. Thus $\Sigma^{\delta} \backslash\left(\left[0, \hat{\mu}^{*}\right] \times I_{\hat{\mu}^{*}}\right)$ is connected (we can also use 
Lemma 3.1 in $\mathrm{BD}$ to reach this conclusion), and its closure contains both $(\lambda / b, 0)$ and $\left(\hat{\mu}^{*}, \bar{U}_{\hat{\mu}^{*}}\right)$. Let $\Sigma_{0}=\Sigma^{\delta} \backslash\left(\left[0, \hat{\mu}^{*}\right] \times I_{\hat{\mu}^{*}}\right)$ and define

$$
\hat{\mu}_{*}=\inf \left\{\mu>0: \text { (3.12) has a positive solution on } \Sigma_{0}\right\} \text {. }
$$

Then $\hat{\mu}_{*} \leq \lambda / b$; thus $\hat{\mu}_{*}<\hat{\mu}^{*}$ and for any $\mu \in\left(\hat{\mu}_{*}, \hat{\mu}^{*}\right)$, (3.12) has a second positive solution other than $\bar{U}_{\mu}$. This completes the proof of Part 5 .

For Part 6, we only need to show that (3.12) has at least two positive solutions on $\Sigma_{0}$ for $\mu \in\left(\hat{\mu}_{*}, \lambda / b\right)$ when $m<m_{0}$. From the bifurcation analysis in subsection 3.1 , the bifurcation at $(\lambda / b, 0)$ is subcritical if $m<m_{0}$; thus $\hat{\mu}_{*}<\lambda / b$. First, similar to the above, we can show that (3.12) still has a positive solution in $\Sigma_{0}$ at $\hat{\mu}_{*}$. This also shows that $\hat{\mu}_{*}>0$ since (3.12) has a unique positive solution when $\mu=0$. Similar to the proof for maximal solutions, we can show that all the minimal solutions with $\mu \geq \hat{\mu}_{*}$ are on $\Sigma_{0}$, and $\Sigma_{0} \cap\left(\left[\hat{\mu}_{*}, \lambda / b\right) \times \widetilde{I}_{\hat{\mu}_{*}}\right)=\left\{\left(\hat{\mu}_{*}, \underline{U}_{\hat{\mu}_{*}}\right)\right\}$, where

$$
\widetilde{I}_{\hat{\mu}_{*}}=\left\{u \in C(\bar{\Omega}): 0 \leq u(x) \leq \underline{U}_{\hat{\mu}_{*}}(x)\right\} .
$$

From the connectedness of $\Sigma_{0}, \Sigma_{0} \backslash\left(\left[\hat{\mu}_{*}, \lambda / b\right) \times \widetilde{I}_{\hat{\mu}_{*}}\right)$ is connected, and its closure contains both $\left(\hat{\mu}_{*}, \underline{U}_{\hat{\mu}_{*}}\right)$ and $\left(\hat{\mu}^{*}, \bar{U}_{\hat{\mu}^{*}}\right)$. Thus (3.12) has at least two solutions on $\Sigma_{0}$ for $\mu \in\left(\hat{\mu}_{*}, \lambda / b\right)$. Moreover, when $\mu \rightarrow(\lambda / b)^{-}, \underline{U}_{\mu}$ is necessarily the solution bifurcating from the zero solution. This proves Part 6 .

In Proposition 3.3, it is assumed that $\hat{\mu}^{*}>\lambda / b$ in Parts 5 and 6. One condition to guarantee that is $m>m_{0}$. Here we give a more precise estimate of $\hat{\mu}^{*}$ in terms of the parameter $m$. To emphasize the dependence of $\hat{\mu}^{*}$ and $\hat{\mu}_{*}$ on $m$, we denote them by $\hat{\mu}^{*}(m)$ and $\hat{\mu}_{*}(m)$, respectively.

Proposition 3.4. Let $M_{a}=\max _{x \in \bar{\Omega}} a(x)$ and $\bar{a}=|\Omega|^{-1} \int_{\Omega} a(x) d x$. Then

(1) $\hat{\mu}^{*}(m) \geq \frac{\lambda M_{a}+m \lambda^{2}}{4 b M_{a}}$, and in particular, $\lim _{m \rightarrow \infty} \hat{\mu}^{*}(m)=\infty$,

(2) $\hat{\mu}^{*}(m)>\lambda / b$ if $m>3 M_{a} / \lambda ; \hat{\mu}_{*}(m)<\lambda / b$ if $m<\frac{\bar{a}}{\lambda}$.

Proof. The function $u_{\lambda}$ is always a supersolution of (3.12) when $\mu>0$; thus we only need to find a positive subsolution lying below $u_{\lambda}$. Then there exists a positive solution of (3.12) for that $\mu$. The constant function $u=k$ is a subsolution if

$$
\lambda-\frac{b \mu}{1+m k}-a(x) k \geq 0, \quad x \in \bar{\Omega},
$$

which is satisfied if

$$
k=\frac{\lambda}{2 M_{a}} \text { and } \mu<\frac{\lambda}{b} \cdot \frac{2 M_{a}+m \lambda}{4 M_{a}} .
$$

Since $u_{\lambda} \geq \frac{\lambda}{M_{a}}$, we thus deduce $\hat{\mu}^{*}(m) \geq \frac{\lambda M_{a}+m \lambda^{2}}{4 b M_{a}}$. Now Part 2 is a simple consequence of Part 1 and the fact that $m_{0}=\bar{a} / \lambda$ (note that solutions of (3.12) are solutions of (2.2) with $c=0)$.

Though Proposition 3.4 gives better bounds of $\hat{\mu}^{*}(m)$ and $\hat{\mu}_{*}(m)$, it is difficult to see from these bounds whether it is possible to have $\hat{\mu}^{*}(m)>\lambda / b>\hat{\mu}_{*}(m)$, which suggests a reversed $S$-shaped bifurcation diagram as in Figure 2 (b). We will show that this is possible if we choose $a(x)$ suitably. More precisely, we can construct such an $a(x)$ as follows: we assume that $\lambda>\lambda_{1}^{D}(\Omega)$ and that $m$ is any fixed constant; since $0<\lambda<\lambda_{1}^{D}\left(\Omega_{0}\right)$, there exists a smooth region $\Omega_{2}$ such that $\Omega \supset \Omega_{2} \supset \overline{\Omega_{0}}$ and 


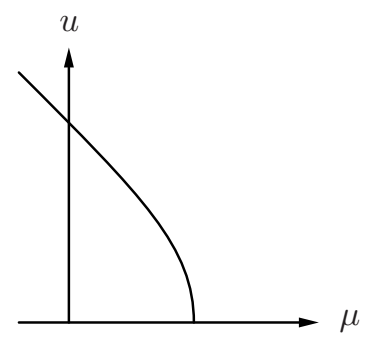

(a)

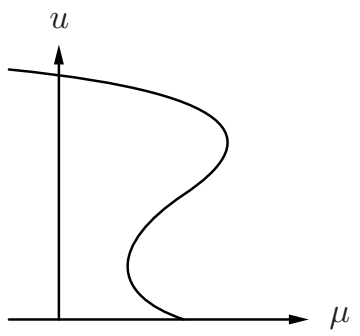

(b)

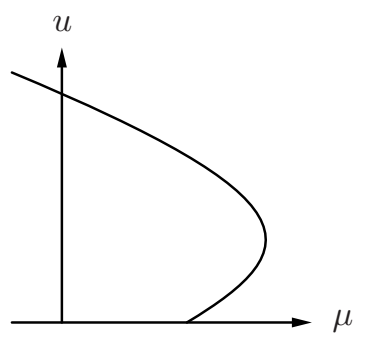

(c)

Figure 2. Possible bifurcation diagrams for (3.12)

$\lambda_{1}^{D}\left(\Omega_{2}\right)<\lambda$, and $\left|\Omega \backslash \Omega_{2}\right|>0$. We define $a_{\varepsilon}(x)=a_{1}(x)+\varepsilon a_{2}(x)$, where $a_{1}(x)>0$ for $x \in \Omega \backslash \overline{\Omega_{2}}$ and $a_{1}(x)=0$ on $\overline{\Omega_{2}}$, while $a_{2}(x)>0$ for $x \in \Omega \backslash \overline{\Omega_{0}}$ and $a_{2}(x)=0$ on $\overline{\Omega_{0}}$. Moreover the value of $a_{1}(x)$ on $\Omega \backslash \overline{\Omega_{2}}$ is chosen so that $\overline{a_{1}}=|\Omega|^{-1} \int_{\Omega} a_{1}(x) d x>\lambda m$. Then for positive $\varepsilon>0, \bar{a}=|\Omega|^{-1} \int_{\Omega} a(x) d x>|\Omega|^{-1} \int_{\Omega} a_{1}(x) d x>\lambda m$. Thus from Proposition 3.4 the bifurcation at $(\mu, u)=(\lambda / b, 0)$ is subcritical. If we can show that (3.12) has a positive solution for some $\mu>\lambda / b$, then $\hat{\mu}^{*}(m)>\lambda / b>\hat{\mu}_{*}(m)$ holds. When $\varepsilon>0$ is small, this can be easily proved by using some results developed in Sections 4 and 5; so we will give a proof of this fact at the end of subsection 5.2. When $\hat{\mu}^{*}(m)>\lambda / b>\hat{\mu}_{*}(m)$ holds, Figure 2 (b) shows a possible bifurcation diagram. Figures 2 (a) and 2 (c) show some other possibilities.

We notice that in (2.2), if $c=0$, then $v(x) \equiv 0$ or $v(x) \equiv \mu$. Thus any positive solution $(u, v)$ satisfies $v=\mu$ and $u$ is a solution of (3.12) when $\mu \geq 0$. Hence Propositions 3.3 and 3.4 give a rather clear description of the set of positive solutions of (2.2) when $c=0$. Now we show that this picture largely remains for positive $c$, in particular for small $c>0$.

Theorem 3.5. Suppose that $0<\lambda<\lambda_{1}^{D}\left(\Omega_{0}\right)$ and that b, c, $m>0$ are fixed. Let $\hat{\mu}^{*}$ and $\hat{\mu}_{*}$ be the critical points defined as in Proposition 3.3 . Then the following hold:

(1) Define

$$
\mu^{*}=\sup \{\mu>0:(2.2) \text { has a positive solution }\}
$$

and

$$
\mu_{*}=\inf \left\{\mu>0:(2.2) \text { has a positive solution }(u, v) \text {, and } u \nsupseteq \bar{U}_{\hat{\mu}^{*}}\right\} \text {. }
$$

Then $\hat{\mu}^{*}-c / m \leq \mu^{*} \leq \hat{\mu}^{*}$ and $\hat{\mu}_{*} \leq \mu_{*} \leq \hat{\mu}_{*}+c / m$.

(2) If $\lambda_{1}^{N}\left(-c p\left(u_{\lambda}\right)\right)<\hat{\mu}^{*}-c / m$, then for $\mu \in\left(\lambda_{1}^{N}\left(-c p\left(u_{\lambda}\right)\right), \hat{\mu}^{*}-c / m\right]$, (2.2) has a positive solution $\left(u_{\mu}^{1}, v_{\mu}^{1}\right)$ satisfying

$$
\begin{aligned}
& \min \left\{\bar{U}_{\mu}(x), \bar{U}_{0}(x)\right\}>u_{\mu}^{1}(x)>\bar{U}_{\mu+c / m}(x), x \in \Omega, \\
& \mu+\frac{c}{m}>v_{\mu}^{1}(x)>\max \{\mu, 0\}, x \in \Omega .
\end{aligned}
$$

(3) If $\hat{\mu}_{*}+c / m<\lambda / b$, then for $\mu \in\left[\hat{\mu}_{*}+c / m, \lambda / b\right)$, (2.2) has a positive solution $\left(u_{\mu}^{2}, v_{\mu}^{2}\right)$ satisfying

$$
\begin{aligned}
& \underline{U}_{\mu}(x)>u_{\mu}^{2}(x)>\max \left\{\underline{U}_{\mu+c / m}(x), 0\right\}, x \in \Omega, \\
& \mu+\frac{c}{m}>v_{\mu}^{2}(x)>\mu, x \in \Omega .
\end{aligned}
$$


(4) If $\hat{\mu}^{*}>\lambda / b+c / m$, then (2.2) has at least two positive solutions for $\lambda / b<$ $\mu \leq \hat{\mu}^{*}-c / m$.

(5) If $\hat{\mu}^{*}>\lambda / b+c / m$ and $\hat{\mu}_{*}<\lambda / b-c / m$, then (2.2) has at least three positive solutions for $\hat{\mu}_{*}+c / m<\mu<\lambda / b$.

All these solutions above can be chosen from the continuum $\Gamma$.

(Notice that $\underline{U}_{\mu+c / m}$ is not always defined in Part 3. In that case we assume $\underline{U}_{\mu+c / m}=0$. Similarly, if $\bar{U}_{\hat{\mu}^{*}}$ is not defined, we understand that it equals 0 .)

Proof. If (2.2) has a solution $(\mu, u, v)$, then from Lemma 3.1 $\mu<v<\mu+c / m$. Thus $u$ is a subsolution of (3.12), and $u_{\lambda}>u$ is always a supersolution of (3.12), so (3.12) has a positive solution for this $\mu$. Hence $\mu^{*} \leq \hat{\mu}^{*}$. Similarly $\hat{\mu}_{*} \leq \mu_{*}$. The proofs of $\hat{\mu}^{*}-c / m \leq \mu^{*}$ and $\mu_{*} \leq \hat{\mu}_{*}+c / m$ are apparent from Parts 2 and 3 to be proved below.

To show the existence of $\left(u_{\mu}^{1}, v_{\mu}^{1}\right)$, we first consider the case of $0<\mu \leq \hat{\mu}^{*}-c / m$. Let $E=C(\bar{\Omega})$, and let $P=\{u \in E: u(x) \geq 0, x \in \bar{\Omega}\}$. Define an order interval in $P^{2} \equiv P \times P$.

$$
J_{1}=\left[\bar{U}_{\mu+c / m}, \bar{U}_{\mu}\right] \times[\mu, \mu+c / m]
$$

where $\left[u_{1}, u_{2}\right]=\left\{u \in E: u_{1}(x) \leq u(x) \leq u_{2}(x)\right\}$. We define an operator

$$
A(u, v)=\left(\begin{array}{c}
(-\Delta+K)^{-1}\left((\lambda+K) u-a(x) u^{2}-\frac{b u v}{1+m u}\right) \\
(-\Delta+K)^{-1}\left((\mu+K) v-v^{2}+\frac{c u v}{1+m u}\right)
\end{array}\right)
$$

where $(u, v) \in J_{1}$, and $K$ is a positive constant. It is easy to see that a fixed point of $A$ is a solution of (2.2). We claim that when $K$ is large enough, then $A(u, v) \in J_{1}$ for each $(u, v) \in J_{1}$. Let $(u, v) \in J_{1}$, and let $A(u, v)=\left(u_{1}, v_{1}\right)$. We choose $K$ large enough so that $f_{K}(x, u, v)=(\lambda+K) u-a(x) u^{2}-b u v /(1+m u)$ is strictly increasing in $u$ and $g_{K}(u, v)=(\mu+K) v-v^{2}+c u v /(1+m u)$ is strictly increasing in $v$, for any $u \in\left[\min _{x} \bar{U}_{\mu+c / m}(x), \max _{x} \bar{U}_{\mu}(x)\right]$ and $v \in[\mu, \mu+c / m]$. Then

$$
\begin{aligned}
u_{1} & =(-\Delta+K)^{-1}\left[(\lambda+K) u-a(x) u^{2}-\frac{b u v}{1+m u}\right] \\
& \geq(-\Delta+K)^{-1}\left[(\lambda+K) \bar{U}_{\mu+c / m}-a(x) \bar{U}_{\mu+c / m}^{2}-\frac{b \bar{U}_{\mu+c / m}}{1+m \bar{U}_{\mu+c / m}}\left(\mu+\frac{c}{m}\right)\right] \\
& =\bar{U}_{\mu+c / m},
\end{aligned}
$$

and similarly,

$$
\begin{aligned}
u_{1} & =(-\Delta+K)^{-1}\left[(\lambda+K) u-a(x) u^{2}-\frac{b u v}{1+m u}\right] \\
& \leq(-\Delta+K)^{-1}\left[(\lambda+K) \bar{U}_{\mu}-a(x) \bar{U}_{\mu}^{2}-\frac{b \bar{U}_{\mu}}{1+m \bar{U}_{\mu}} \mu\right]=\bar{U}_{\mu} .
\end{aligned}
$$


On the other hand,

$$
\begin{aligned}
v_{1} & =(-\Delta+K)^{-1}\left[(\mu+K) v-v^{2}+\frac{c u v}{1+m u}\right] \\
& \geq(-\Delta+K)^{-1}\left[(\mu+K) v-v^{2}\right] \\
& \geq(-\Delta+K)^{-1}(K \mu)=\mu,
\end{aligned}
$$

since the minimum of $f_{1}(v)=(\mu+K) v-v^{2}$ on $[\mu, \mu+c / m]$ is achieved at $v=\mu$; and

$$
\begin{aligned}
v_{1} & =(-\Delta+K)^{-1}\left[(\mu+K) v-v^{2}+\frac{c u v}{1+m u}\right] \\
& <(-\Delta+K)^{-1}\left[(\mu+K) v-v^{2}+\frac{c}{m} v\right] \\
& \leq(-\Delta+K)^{-1}\left[K\left(\mu+\frac{c}{m}\right)\right]=\mu+\frac{c}{m},
\end{aligned}
$$

since the maximum of $f_{2}(v)=(\mu+K+(c / m)) v-v^{2}$ on $[\mu, \mu+c / m]$ is achieved at $v=\mu+c / m$. Therefore $\left(u_{1}, v_{1}\right)=A(u, v) \in J_{1}$. Since $A$ is a compact operator from the convex set $J_{1}$ to $J_{1}, A$ has a fixed point in $J_{1}$ by the Schauder fixed point theorem.

Now we modify the above proof for $\mu \in\left(\lambda_{1}^{N}\left(-c p\left(u_{\lambda}\right)\right), 0\right]$. In this case, we define

$$
J_{2}=\left[\bar{U}_{\mu+c / m}, \bar{U}_{0}\right] \times[0, \mu+c / m] ;
$$

then from the same proof as above, we still have $u_{1} \geq \bar{U}_{\mu}$, and $v_{1} \leq \mu+c / m$. By using $v \geq 0$ and $u \leq \bar{U}_{0}$, we obtain $u_{1} \leq \bar{U}_{0}$. Similarly, by using $v \geq 0$ and the fact that the minimum of $f_{1}(v)=(\mu+K) v-v^{2}$ on $[0, \mu+c / m]$ is achieved at $v=0$, we obtain $v_{1} \geq 0$. Therefore $A$ is invariant on $J_{2}$, and again we have a fixed point of $A$ in $J_{2}$. However, $\left(\bar{U}_{0}, 0\right)$ is already a fixed point of $A$ in $J_{2}$. To show that $A$ has a fixed point in $J_{2}$ with both components positive, we make use of the fixed point index with respect to the convex set $J_{2}$. By definition, $\operatorname{ind}\left(A, J_{2}\right)=1$. Since $\mu>\lambda_{1}^{N}\left(-c p\left(u_{\lambda}\right)\right)$, we find that $\left(\bar{U}_{0}, 0\right)=\left(u_{\lambda}, 0\right)$ is an isolated fixed point of $A$ in $J_{2}$. Moreover, by applying the fixed point index calculation result in [Da1], we find that $\operatorname{ind}\left(A,\left(\bar{U}_{0}, 0\right)\right)=0$; that is, there exists a small neighborhood $N_{0}$ of $\left(\bar{U}_{0}, 0\right)$ in $J_{2}$ such that $\operatorname{ind}\left(A, N_{0}\right)=0$. Therefore, $\operatorname{ind}\left(A, J_{2} \backslash N_{0}\right)=1-0=1$ and $A$ has a fixed point in $J_{2} \backslash N_{0}$, which is necessarily a positive solution of (2.2). This completes the proof of Part 2, and the proof of Part 3 is similar.

We define a subset of $\mathbf{R} \times P^{2}$ for each $\widetilde{\mu} \in\left(\lambda_{1}^{N}\left(-c p\left(u_{\lambda}\right)\right), \hat{\mu}^{*}-c / m\right]$ :

$$
J_{\widetilde{\mu}}=\left[\lambda_{1}^{N}\left(-c p\left(u_{\lambda}\right)\right), \widetilde{\mu}\right] \times\left[\bar{U}_{\widetilde{\mu}+c / m}, \bar{U}_{0}\right] \times[0, \widetilde{\mu}+c / m] .
$$

We notice that $\{\mu\} \times J_{1} \subset J_{\widetilde{\mu}}$ if $\mu \in\left(0, \hat{\mu}^{*}-c / m\right]$ and $\mu \leq \widetilde{\mu}$, and $\{\mu\} \times J_{2} \subset J_{\mu}$ if $\mu \in\left(\lambda_{1}^{N}\left(-c p\left(u_{\lambda}\right)\right), 0\right]$ and $\mu \leq \widetilde{\mu}$. Thus $\left(\mu, u_{\mu}^{1}, v_{\mu}^{1}\right) \in J_{\widetilde{\mu}}$ for $\lambda_{1}^{N}\left(-c p\left(u_{\lambda}\right)\right)<\mu \leq \widetilde{\mu}$. If $(\mu, u, v) \in \partial J_{\widetilde{\mu}}$ and $\mu \in\left(\lambda_{1}^{N}\left(-c p\left(u_{\lambda}\right)\right), \widetilde{\mu}\right)$, then from the above calculation of $\left(u_{1}, v_{1}\right)=A(u, v)$, we can see that either $\left(\mu, u_{1}, v_{1}\right)=\left(\mu, \bar{U}_{0}, 0\right)$ or $\left(\mu, u_{1}, v_{1}\right) \in$ $\operatorname{int}\left(J_{\widetilde{\mu}}\right)$. Hence $(\mu, u, v)$ cannot be a positive fixed point of $A$.

Let $\Gamma$ be the continuum of positive solutions of (2.2) obtained in Theorem 3.2 . Then $\Gamma \cap J_{\widetilde{\mu}} \neq \emptyset$ since the bifurcation at $\left(\lambda_{1}^{N}\left(-c p\left(u_{\lambda}\right)\right), \bar{U}_{0}, 0\right)$ is supercritical. From the arguments above, if $(\mu, u, v) \in \Gamma \cap \partial J_{\widetilde{\mu}}$, then either $\mu=\lambda_{1}^{N}\left(-c p\left(u_{\lambda}\right)\right)$ or $\mu=\widetilde{\mu}$. The latter case must happen since (2.2) has no positive solution when $\mu=\lambda_{1}^{N}\left(-c p\left(u_{\lambda}\right)\right)$. From the connectedness of $\Gamma$, we see that $\Gamma \cap \partial J_{\hat{\mu}^{*}-c / m} \neq$ $\emptyset$. Therefore, $\Gamma \backslash J_{\hat{\mu}^{*}-c / m}$ has a component connecting $(\lambda / b, 0, \lambda / b)$ to a solution 
$(\mu, u, v) \in \Gamma \cap \partial J_{\hat{\mu}^{*}-c / m}$, and by the above discussion, we must have $\mu=\hat{\mu}^{*}-c / m$. Therefore (2.2) has at least one positive solution for $\lambda / b<\mu \leq \hat{\mu}^{*}-c / m$ which is on $\Gamma \backslash J_{\hat{\mu}^{*}-c / m}$. This proves Part 4.

For Part 5, we use the minimal positive solutions to define a set $J$ similar to $J_{\hat{\mu}^{*}-2 / m}$ and then show that $\Gamma \backslash J_{\hat{\mu}^{*}-2 / m}$ contains a positive solution in $J$ and another one outside $J$. Since the arguments are analogous, we omit the details.

3.3. Dynamical behavior. First we consider the dynamics of the auxiliary equation:

$$
\begin{cases}u_{t}-\Delta u=\lambda u-a(x) u^{2}-b \mu \frac{u}{1+m u}, & x \in \Omega, t>0, \\ \partial_{\nu} u=0, & x \in \partial \Omega, t>0, \\ u(x, 0)=u_{0}(x) \geq 0, & x \in \Omega .\end{cases}
$$

Theorem 3.6. Suppose that $0<\lambda<\lambda_{1}^{D}\left(\Omega_{0}\right)$ and that $b, c, m>0$ are fixed. Then all solutions $u(x, t)$ of (3.29) are globally bounded, and the following hold:

(1) If $\mu \leq 0$, then $\bar{U}_{\mu}$ is globally asymptotically stable.

(2) If $\mu>\hat{\mu}^{*}$, then 0 is globally asymptotically stable.

(3) If $0<\mu<\lambda / b$, then for any $u_{0}, \underline{\lim }_{t \rightarrow \infty} u(x, t) \geq \underline{U}_{\mu}(x)$.

(4) If $0<\mu \leq \hat{\mu}^{*}$, then for any $u_{0}, \overline{\lim }_{t \rightarrow \infty} u(x, t) \leq \bar{U}_{\mu}(x)$, and if $u_{0}(x) \geq$ $\bar{U}_{\mu}(x)$, then $\lim _{t \rightarrow \infty} u(x, t)=\bar{U}_{\mu}(x)$; if $u_{0}(x) \geq \bar{U}_{\hat{\mu}^{*}}(x)$, then

$$
V_{\mu, 1}(x) \leq \underline{\lim }_{t \rightarrow \infty} u(x, t) \leq \varlimsup_{\lim }^{t \rightarrow \infty} u(x, t) \leq \bar{U}_{\mu}(x),
$$

where $V_{\mu, 1}(x)$ is the unique positive solution of

$$
-\Delta u=\left(\lambda-\frac{b \mu}{1+m U}\right) u-a(x) u^{2}, x \in \Omega, \partial_{\nu} u=0, x \in \partial \Omega,
$$

with $U=\bar{U}_{\hat{\mu}^{*}}$.

(5) If $\hat{\mu}_{*}<\lambda / b, \hat{\mu}_{*}<\mu<\lambda / b$, and $u_{0}(x) \leq \underline{U}_{\mu}(x)$, then

$$
\lim _{t \rightarrow \infty} u(x, t)=\underline{U}_{\mu}(x)
$$

$$
\text { if } \begin{aligned}
u_{0}(x) & \leq \underline{U}_{\hat{\mu}_{*}}(x) \text {, then } \\
& \underline{U}_{\mu}(x) \leq \underline{\lim }_{t \rightarrow \infty} u(x, t) \leq \varlimsup_{t \rightarrow \infty} u(x, t) \leq V_{\mu, 2}(x),
\end{aligned}
$$

where $V_{\mu, 2}$ is the unique positive solution of (3.31) with $U=\underline{U}_{\hat{\mu}_{*}}$.

(6) If $\hat{\mu}_{*}<\lambda / b$, then there exists $\hat{\mu}^{\sharp} \in\left(\lambda / b, \hat{\mu}^{*}\right)$ such that $\lambda=$ $\lambda_{1}^{N}\left(b \hat{\mu}^{\sharp} /\left(1+m \underline{U}_{\hat{\mu}_{*}}\right)\right)$, and for $\lambda / b \leq \mu<\hat{\mu}^{\sharp}$, if $u_{0}(x) \leq \underline{U}_{\hat{\mu}_{*}}(x)$, then

$$
0 \leq \underline{\lim }_{t \rightarrow \infty} u(x, t) \leq \varlimsup_{t \rightarrow \infty} u(x, t) \leq V_{\mu, 2}(x) .
$$

For $\mu \geq \hat{\mu}^{\sharp}$, if $u_{0}(x) \leq \underline{U}_{\hat{\mu}_{*}}(x)$, then $\lim _{t \rightarrow \infty} u(x, t)=0$.

Proof. For Part 1, the global asymptotical stability of $\bar{U}_{\mu}$ is well known since the nonlinearity is concave. For $\mu>0$, from Theorem 2.3 , $u_{\lambda}$ is globally asymptotically stable for (2.4). Let $u_{1}(x, t)$ be the solution of (2.4) with the same initial value $u_{0}$; then $u_{1}(x, t)>u(x, t)$ for $t>0$ if $\mu>0$. On the other hand, from the maximum principle $u(x, t) \geq 0$ for all $t \geq 0$. Thus $u(x, t)$ is globally bounded in $C(\bar{\Omega})$, and $\varlimsup_{t \rightarrow \infty} u(x, t) \leq u_{\lambda}(x)$ if $\mu>0$.

It is well known that the $\omega$-limit set of $\{u(\cdot, t)\}$ is the union of the steady state solutions. Thus in Part 2, the unique steady state must be globally asymptotically stable. For Part 3, since the trivial steady-state $u=0$ is linearly unstable for 
$\mu<\lambda / b$, the $\omega$-limit set of $u(\cdot, t)$ can only consist of positive steady state solutions, which implies $\underline{\lim }_{t \rightarrow \infty} u(x, t) \geq \underline{U}_{\mu}$. The first conclusion in Part 4 also follows from this line of reasoning. For the other conclusions in Part 4 , we compare $u(x, t)$ with the solution $u_{2}(x, t)$ of

$$
\begin{cases}u_{t}-\Delta u=\left(\lambda-\frac{b \mu}{1+m U}\right) u-a(x) u^{2}, & x \in \Omega, t>0, \\ \partial_{\nu} u=0, & x \in \partial \Omega, t>0, \\ u(x, 0)=U(x) \geq 0, & x \in \Omega,\end{cases}
$$

with $U=\bar{U}_{\hat{\mu}^{*}}$. Then $u(x, t)>u_{2}(x, t) \geq U$ for $t>0$. Hence (3.30) can be obtained since $\lim _{t \rightarrow \infty} u_{2}(x, t)=V_{\mu, 1}(x)$. If $u_{0} \geq \bar{U}_{\mu}$, then $u(x, t) \geq \bar{U}_{\mu}$ for all $t>0$ and hence, by (3.30), $u(x, t) \rightarrow \bar{U}_{\mu}$ as $t \rightarrow \infty$. The proof of Part 5 is similar. For Part 6 , notice that $\hat{\mu}^{\sharp}$ is the critical value so that $V_{\mu, 2}$ does not exist if $\mu \geq \hat{\mu}^{\sharp}$. Then the proof is also similar to that of Part 4.

Remark 3.7. (1) Similar to (2.5), (3.31) has a unique positive solution $V_{\mu}(x)$ which is strictly decreasing in $\mu$ for any given $U$ as long as $\lambda_{1}^{N}(\lambda-b \mu /(1+m U))=$ $\lambda-\lambda_{1}^{N}(b \mu /(1+m U))>0$. Moreover, $V_{\mu}$ is globally asymptotically stable as a steady state solution of (3.34), and $\lim _{\mu \rightarrow 0^{+}} V_{\mu, 1}=u_{\lambda}, V_{\hat{\mu}^{*}, 1}=\bar{U}_{\hat{\mu}^{*}}$. Similar remarks apply to $V_{\mu, 2}$.

(2) Since the right hand side of (3.29) is analytic in $u$ for nonnegative $u$, by a well-known result of L. Simon [Si], any solution of (3.29) converges to a single steady state solution as $t \rightarrow \infty$. Therefore some of the statements in Theorem 3.6 can be improved.

Now we turn to the dynamics of (2.1).

Theorem 3.8. Suppose that $0<\lambda<\lambda_{1}^{D}\left(\Omega_{0}\right)$ and that $b, c, m>0$ are fixed. Then all solutions $(u(x, t), v(x, t))$ of (2.1) are globally bounded, and $v(x, t)$ satisfies

$$
\max \{\mu, 0\} \leq \underline{\lim }_{t \rightarrow \infty} v(x, t) \leq \varlimsup_{t \rightarrow \infty} v(x, t) \leq \max \left\{\mu+\frac{c}{m}, 0\right\},
$$

and the asymptotic behavior of $u(x, t)$ is as follows:

(1) If $\mu<\lambda_{1}^{N}\left(-c p\left(u_{\lambda}\right)\right)$, then $\lim _{t \rightarrow \infty} u(x, t)=u_{\lambda}(x)$ and $\lim _{t \rightarrow \infty} v(x, t)=0$ uniformly for $x \in \bar{\Omega}$.

(2) If $\mu>\hat{\mu}^{*}$, then $\lim _{t \rightarrow \infty} u(x, t)=0$ and $\lim _{t \rightarrow \infty} v(x, t)=\mu$ uniformly for $x \in \bar{\Omega}$.

(3) If $\lambda_{1}^{N}\left(-c p\left(u_{\lambda}\right)\right)<\mu<\hat{\mu}^{*}-c / m$, and $u_{0}(x) \geq \bar{U}_{\hat{\mu}^{*}}(x)$, then

$$
V_{\mu+c / m, 1}(x) \leq \underline{\lim }_{t \rightarrow \infty} u(x, t) \leq \varlimsup_{\lim } \rightarrow \infty u(x, t) \leq \min \left\{\bar{U}_{\mu}(x), \bar{U}_{0}(x)\right\},
$$
where $V_{\mu, 1}$ is defined in Theorem 3.6.

(4) Suppose that $\hat{\mu}_{*}<\lambda / b$. Let $\hat{\mu}^{\sharp}$ be the constant defined in Theorem 3.6 . Then for $\hat{\mu}_{*}+c / m \leq \mu<\hat{\mu}^{\sharp}$, if $u_{0}(x) \leq \underline{U}_{\hat{\mu}_{*}}(x)$, then

$$
\max \left\{\underline{U}_{\mu+c / m}(x), 0\right\} \leq \underline{\lim }_{t \rightarrow \infty} u(x, t) \leq \varlimsup_{\lim _{t \rightarrow \infty}} u(x, t) \leq V_{\mu, 2}(x),
$$

where $V_{\mu, 2}$ is defined in Theorem [3.6, and for $\mu \geq \hat{\mu}^{\sharp}$, if $u_{0}(x) \leq \underline{U}_{\hat{\mu}_{*}}(x)$, then $\lim _{t \rightarrow \infty} u(x, t)=0$ and $\lim _{t \rightarrow \infty} v(x, t)=\mu$ uniformly for $x \in \bar{\Omega}$.

Proof. By the comparison principle, we have $0 \leq \underline{\lim }_{t \rightarrow \infty} u(x, t) \leq \overline{\lim }_{t \rightarrow \infty} u(x, t) \leq$ $u_{\lambda}(x)$ and $\mu \leq \underline{\lim }_{t \rightarrow \infty} v(x, t) \leq \varlimsup_{t \rightarrow \infty} v(x, t) \leq \mu+c / m$. Thus all solutions are globally bounded. 
We prove Part 1. If $\mu<\lambda_{1}^{N}\left(-c p\left(u_{\lambda}\right)\right)$, then there exists $\delta_{1}>0$ such that $\lambda_{1}^{N}\left(\mu+c p\left(u_{\lambda}+\delta_{1}\right)\right)<0$. Since $\varlimsup_{t \rightarrow \infty} u(x, t) \leq u_{\lambda}(x)$, there exists $T_{1}>0$ such that when $t \geq T_{1}$, then $u(x, t) \leq u_{\lambda}(x)+\delta_{1}$. Then

$$
v_{t}-\Delta v=\mu v-v^{2}+\frac{c u v}{1+m u} \leq\left(\mu+c p\left(u_{\lambda}+\delta_{1}\right)\right) v-v^{2}, \quad t \geq T_{1} .
$$

Since $\lambda_{1}^{N}\left(\mu+c p\left(u_{\lambda}+\delta_{1}\right)\right)<0$, then $v=0$ is globally asymptotically stable for the dynamics of $v_{t}-\Delta v=\left(\mu+c p\left(u_{\lambda}+\delta_{1}\right)\right) v-v^{2}$ with Neumann boundary condition. Thus $v(x, t) \rightarrow 0$ as $t \rightarrow \infty$. Hence for any small $\delta_{2}>0$ which satisfies $\lambda-b \delta_{2}>0$, there exists $T_{2}>T_{1}$ such that $v(x, t) \leq \delta_{2}$ for $t \geq T_{2}$. Then

$u_{t}-\Delta u=\lambda u-a(x) u^{2}-\frac{b u v}{1+m u} \geq \lambda u-a(x) u^{2}-b \delta_{2} p(u) \geq\left(\lambda-b \delta_{2}\right) u-a(x) u^{2}$.

Since $\lambda-b \delta_{2}>0$, then $u=u_{\lambda-b \delta_{2}}$ is globally asymptotically stable for the dynamics of $u_{t}-\Delta u=\left(\lambda-b \delta_{2}\right) u-a(x) u^{2}$ with Neumann boundary condition. Thus $\varliminf_{t \rightarrow \infty} u(x, t) \geq u_{\lambda-b \delta_{2}}(x)$. As $\delta_{2} \rightarrow 0$, we obtain $\lim _{t \rightarrow \infty} u(x, t)=u_{\lambda}(x)$. The proof of Part 2 is similar since $u=0$ is globally asymptotically stable for the dynamics of (3.29).

Next we prove Part 3. By the estimate for $v(x, t)$ in (3.35), for any $\delta_{3}>0$, there exists $T_{3}>0$ such that for $t>T_{3}$,

$$
\max \{0, \mu\}-\delta_{3}<v(x, t)<\max \left\{0, \mu+\frac{c}{m}\right\}+\delta_{3} .
$$

Then the estimate for $u(x, t)$ can be obtained by using Part 4 of Theorem 3.6 and letting $\delta_{3} \rightarrow 0$. The proof of Part 4 is similar.

3.4. Discussion. To conclude this section, we discuss some biological implications of Theorem 3.8. In this section the prey has a weak growth rate $\lambda<\lambda_{1}^{D}\left(\Omega_{0}\right)$. The main impact of the weak prey growth rate is that both populations stay bounded, and the dynamics of (2.1) has three possibilities according to the predator growth rate $\mu$ :

(1) (Weak predator growth rate) When $\mu<\lambda_{1}^{N}\left(-c p\left(u_{\lambda}\right)\right)<0$, the predator will become extinct, while the prey will reach its carrying capacity. Thus we have unconditional persistence for the prey and unconditional extinction for the predator.

(2) (Strong predator growth rate) This is when $\mu>\hat{\mu}^{*}$. Opposite to the previous case, the prey will become extinct while the predator will persist unconditionally for any initial population distribution.

(3) (Intermediate predator growth rate) If $\hat{\mu}_{*}+c / m<\mu<\hat{\mu}^{*}-c / m$ with all these constants well defined and well ordered, then a bistable phenomenon appears, where two attracting regions exist: one is defined in (3.36) for $u$ and (3.35) for $v$, and the other is defined in (3.37) for $u$ and (3.35) for $v$. The former one contains a coexistence steady state, but it is unclear whether that coexistence steady state is the global attractor; the latter attracting region contains a coexistence steady state with a smaller $u$ component when $\hat{\mu}_{*}+c / m<\mu<\lambda / b$, but that coexistence steady state becomes $(0, \mu)$ (prey extinction and predator persistence) when $\mu>\hat{\mu}^{\sharp}$. Though the predator population will settle in between $\mu$ and $\mu+c / m$, the fate of the prey will depend on its initial population. We notice that it is possible that $\hat{\mu}_{*}=$ $\hat{\mu}^{*}=\lambda / b$. Then a coexistence steady state exists for $\lambda_{1}^{N}\left(-c p\left(u_{\lambda}\right)\right)<\mu<$ 
$\lambda / b$, and this is a case in which the system exhibits permanence. However, our discussion before Theorem 3.5 shows that bistability can happen for certain ranges of the parameters if $a(x)$ is chosen properly.

Thus Theorem 3.8 gives some criteria of persistence or extinction of the prey population. Other criteria will be discussed in the next section, and some of them can also be adapted to the weak prey growth case (see Theorem 4.11).

\section{Strong PREY Growth Rate}

In this section we assume that $\lambda>\lambda_{1}^{D}\left(\Omega_{0}\right)$, and we will consider the structure of the set of nonnegative steady state solutions of (2.1) and the underlying dynamical behavior.

4.1. Steady state solutions. Compared to the weak prey growth case, there is only one semi-trivial branch $\Gamma_{v}$, and there is still a bifurcation point $\mu_{2}=\lambda / b$ such that a smooth curve of positive solutions $\Gamma_{2}^{\prime}$ (contained in a global branch $\Gamma_{2}$ ) to (2.2) bifurcates from $\Gamma_{v}$ at $(\mu, u, v)=\left(\mu_{2}, 0, \mu_{2}\right)$. Since this is the only possible bifurcation point for positive solutions, $\Gamma_{2}$ is unbounded in the space of $(\mu, u, v)$. Using Lemma 3.1 we can still show that $\mu>-c / m$ for any positive solution $(\mu, u, v)$. Thus if all the positive solutions $(u, v)$ of (2.2) are a priori bounded for bounded $\mu$, then

$$
\operatorname{proj}_{\mu} \Gamma_{2} \supset(\lambda / b, \infty) .
$$

Here we prove that such an a priori bound does exist.

Proposition 4.1. Let $\lambda>\lambda_{1}^{D}\left(\Omega_{0}\right)$ be fixed and $\mu_{n} \leq M$. Then there exists a positive constant $C$ independent of $n$ such that any positive solution $\left(u_{n}, v_{n}\right)$ of (2.2) (with $\mu=\mu_{n}$ ) satisfies

$$
\left\|u_{n}\right\|_{\infty}+\left\|v_{n}\right\|_{\infty} \leq C .
$$

Proof. Since $\mu_{n} \leq M$, we have

$$
-\Delta v_{n} \leq\left(M+\frac{c}{m}\right) v_{n}-v_{n}^{2} .
$$

Thus $\left\|v_{n}\right\|_{\infty} \leq M+c / m$. Suppose that the proposition is not true; then there exists a sequence $\left\{\left(u_{n}, v_{n}\right)\right\}$ satisfying (2.2) with $\mu=\mu_{n}$ such that $\left\|u_{n}\right\|_{\infty} \rightarrow \infty$ as $n \rightarrow \infty$.

Denote $\widehat{u_{n}}=u_{n} /\left\|u_{n}\right\|_{\infty}$. Then $-\Delta \widehat{u_{n}} \leq \lambda \widehat{u_{n}}$, and

$$
\int_{\Omega}\left|\nabla \widehat{u_{n}}\right|^{2} d x+\int_{\Omega}{\widehat{u_{n}}}^{2} d x \leq(\lambda+1) \int_{\Omega}{\widehat{u_{n}}}^{2} d x \leq(\lambda+1)|\Omega| .
$$

Hence $\left\{\widehat{u_{n}}\right\}$ is bounded in $H^{1}(\Omega)$, and there is a subsequence (which we still denote by $\left.\left\{\widehat{u_{n}}\right\}\right)$ converging to some $\widehat{u}$ weakly in $H^{1}(\Omega)$ and strongly in $L^{2}(\Omega)$. Since $\left\|\widehat{u_{n}}\right\|_{\infty}=1$, we can also assume that $\widehat{u_{n}} \rightarrow \widehat{u}$ in $L^{p}(\Omega)$ for any $p>1$, and $0 \leq \widehat{u} \leq 1$. We claim that $\widehat{u} \not \equiv 0$. Suppose that $\widehat{u} \equiv 0$. Then $\widehat{u_{n}} \rightarrow 0$ in $L^{p}(\Omega)$ for any $p>1$, and from elliptic regularity, $(-\Delta+I)^{-1} \widehat{u_{n}} \rightarrow 0$ in $C^{1}(\bar{\Omega})$. But on the other hand, $(-\Delta+I) \widehat{u_{n}} \leq(\lambda+1) \widehat{u_{n}}$; thus $0 \leq \widehat{u_{n}} \leq(\lambda+1)(-\Delta+I)^{-1} \widehat{u_{n}}$, and it follows that $\widehat{u_{n}} \rightarrow 0$ in $L^{\infty}(\Omega)$. That is a contradiction with $\left\|\widehat{u_{n}}\right\|_{\infty}=1$. Therefore $\widehat{u} \not \equiv 0$. Similarly we define $\widehat{v_{n}}=v_{n} /\left\|v_{n}\right\|_{\infty}$; then subject to a subsequence, $\widehat{v_{n}} \rightarrow \widehat{v}$ weakly in $H^{1}(\Omega)$ and strongly in $L^{p}(\Omega)$ for any $p>1$, and $\widehat{v} \not \equiv 0$. 
Moreover, $\widehat{u_{n}}$ satisfies

$$
-\Delta \widehat{u_{n}}=\lambda \widehat{u_{n}}-a(x)\left\|u_{n}\right\|_{\infty}{\widehat{u_{n}}}^{2}-\frac{b \widehat{u_{n}}}{1+m\left\|u_{n}\right\|_{\infty} \widehat{u_{n}}} v_{n}
$$

We multiply this equation by a smooth function $\phi$ whose support is in $\Omega_{0}$, and integrate over $\Omega_{0}$. Then

$$
\int_{\Omega_{0}} \nabla \widehat{u_{n}} \nabla \phi d x=\lambda \int_{\Omega_{0}} \widehat{u_{n}} \phi d x-\int_{\Omega_{0}} \frac{b \widehat{u_{n}}}{1+m\left\|u_{n}\right\|_{\infty} \widehat{u_{n}}} v_{n} \phi d x .
$$

For the last integral,

$$
\left|\int_{\Omega_{0}} \frac{\widehat{u_{n}}}{1+m|| u_{n} \|_{\infty} \widehat{u_{n}}} v_{n} \phi d x\right| \leq \frac{\left\|v_{n}\right\|_{\infty}}{m\left\|u_{n}\right\|_{\infty}}\|\phi\|_{L^{1}(\Omega)} \rightarrow 0, \quad \text { as } n \rightarrow \infty .
$$

Hence when $n \rightarrow \infty$, we have

$$
\int_{\Omega_{0}} \nabla \widehat{u} \nabla \phi d x=\lambda \int_{\Omega_{0}} \widehat{u} \phi d x
$$

which implies that $\widehat{u} \geq 0$ is a weak solution of

$$
-\Delta \widehat{u}=\lambda \widehat{u}, \quad x \in \Omega_{0} .
$$

From the maximum principle, either $\widehat{u}>0$ in $\Omega_{0}$ or $\widehat{u} \equiv 0$ in $\Omega_{0}$. But if the former is true, then from a well-known variant of the maximum principle (see [BNV]), we have $\lambda_{1}^{D}\left(-\lambda, \Omega_{0}\right) \geq 0$, which contradicts the fact that $\lambda_{1}^{D}\left(-\lambda, \Omega_{0}\right)=-\lambda+\lambda_{1}^{D}\left(0, \Omega_{0}\right)<0$ from the assumption. Hence we must have $\widehat{u} \equiv 0$ in $\bar{\Omega}_{0}$.

Next we multiply (4.5) by $\left(1+m\left\|u_{n}\right\|_{\infty} \widehat{u_{n}}\right) /\left\|u_{n}\right\|_{\infty}^{2}$ and integrate over $\Omega$. Then

$$
\begin{aligned}
\frac{m}{\left\|u_{n}\right\|_{\infty}} \int_{\Omega}\left|\nabla \widehat{u_{n}}\right|^{2} d x= & \frac{\lambda}{\left\|u_{n}\right\|_{\infty}^{2}} \int_{\Omega} \widehat{u_{n}} d x+\frac{\lambda m}{\left\|u_{n}\right\|_{\infty}} \int_{\Omega}{\widehat{u_{n}}}^{2} d x \\
& -\frac{1}{\left\|u_{n}\right\|_{\infty}} \int_{\Omega} a(x){\widehat{u_{n}}}^{2} d x-m \int_{\Omega} a(x){\widehat{u_{n}}}^{3} d x \\
& -\frac{b}{\left\|u_{n}\right\|_{\infty}^{2}} \int_{\Omega} \widehat{u_{n}} v_{n} d x .
\end{aligned}
$$

When $n \rightarrow \infty$, we obtain

$$
0=-m \int_{\Omega \backslash \overline{\Omega_{0}}} a(x) \widehat{u}^{3} d x .
$$

Since $a(x)>0$ on $\Omega \backslash \overline{\Omega_{0}}$, we find $\widehat{u}=0$ almost everywhere in $\Omega \backslash \overline{\Omega_{0}}$. Combined with the arguments in the last paragraph, we have $\widehat{u} \equiv 0$ in $\bar{\Omega}$, which is a contradiction. Therefore (4.2) holds.

Combining the bifurcation analysis and the above a priori estimate, we have

Theorem 4.2. Suppose that $\lambda>\lambda_{1}^{D}\left(\Omega_{0}\right)$ is fixed. Then there exists a continuum $\Gamma$ of positive solutions of (2.2) such that

$$
\operatorname{proj}_{\mu} \Gamma=\left[\mu_{*}, \infty\right) \text { or }\left(\mu_{*}, \infty\right), \quad-\frac{c}{m}<\mu_{*} \leq \frac{\lambda}{b}
$$

and satisfies the following:

(1) $\Gamma$ bifurcates from $(\mu, u, v)=(\lambda / b, 0, \lambda / b)$, and the bifurcation of $\Gamma$ at $(\lambda / b, 0$, $\lambda / b)$ is supercritical $\left(\mu_{2}^{\prime}(0)>0\right)$ if $m>m_{0}$ and it is subcritical $\left(\mu_{2}^{\prime}(0)<0\right)$ if $0 \leq m<m_{0}$, where $m_{0}$ is defined by (3.11).

(2) For any $\mu>\mu_{*}$, (2.2) has at least one positive solution. 
(3) If $\mu_{*}<\lambda / b$, then for $\mu=\mu_{*}$, (2.2) has a positive solution, and thus $\operatorname{proj}_{\mu} \Gamma=\left[\mu_{*}, \infty\right)$.

(4) All these solutions mentioned above can be chosen from the unbounded continuum $\Gamma$.

Proof. The local bifurcation analysis is the same as that in subsection 3.1, and from Proposition 4.1, we obtain (4.12). The proof for the other parts is standard and hence is omitted.

4.2. The scalar equation and its perturbation. A better understanding of the set of steady state solutions and the dynamics of the system (2.1) will rely on a better understanding of the scalar equation (3.12). In this subsection we will consider the scalar equation (3.12) again, but now we assume $\lambda>\lambda_{1}^{D}\left(\Omega_{0}\right)$. As can be seen from our next result, the behavior of the global bifurcation branch of the positive solutions of (3.12) is now drastically different from that for the case $\lambda<\lambda_{1}^{D}\left(\Omega_{0}\right)$ discussed in Proposition 3.3 ,

Proposition 4.3. Suppose that $\lambda>\lambda_{1}^{D}\left(\Omega_{0}\right)$ and that $b, m>0$ are fixed. Then $\mu=\lambda / b$ is a bifurcation point for (3.12) such that a global continuum $\Sigma_{0}$ of positive solutions of (3.12) emanates from $(\mu, u)=(\lambda / b, 0)$, and

$$
\operatorname{proj}_{\mu} \Sigma_{0}=\left[\mu_{*}^{0}, \infty\right) \text { or }\left(\mu_{*}^{0}, \infty\right), \quad 0<\mu_{*}^{0} \leq \frac{\lambda}{b} .
$$

Moreover the conclusions in Parts 1-4 of Theorem 4.2 hold with $\Gamma$ replaced by $\Sigma_{0}$ and $\mu_{*}$ replaced by $\mu_{*}^{0}$. Furthermore, the following hold:

(1) $\mu_{*}^{0}=\inf \{\mu:$ (3.12) has a positive solution $\}$.

(2) If $\mu_{*}^{0}<\lambda / b$, then for $\mu \in\left[\mu_{*}^{0}, \lambda / b\right)$, (2.2) has a minimal solution $\underline{U}_{\mu}$, which is strictly decreasing with respect to $\mu$, and for $\mu \in\left(\mu_{*}^{0}, \lambda / b\right)$, (2.2) has at least two positive solutions.

Proof. The conclusions in the first half directly follow from Theorem 4.2 by letting $c=0$, since in this case the equation of $v$ becomes $-\Delta v=\mu v-v^{2}$, and the equation of $u$ becomes (3.12) with $v=\mu$, and the a priori estimates in Proposition 4.1 still hold. Parts 1 and 2 can be proved as in the proof of Proposition 3.3, since (3.12) has the monotonicity property.

The next proposition gives the asymptotic limit of the positive solutions of (3.12) as $\mu \rightarrow \infty$, which will be used in later discussions.

Proposition 4.4. Suppose that $\lambda>\lambda_{1}^{D}\left(\Omega_{0}\right)$ and that $b, m>0$ are fixed. Let $\left(\mu_{n}, u_{n}\right)$ be a sequence of positive solutions of (3.12) and $\mu_{n} \rightarrow \infty$ as $n \rightarrow \infty$. Then subject to a subsequence,

$$
\lim _{n \rightarrow \infty} \mu_{n}\left\|u_{n}\right\|_{\infty}^{-1}=\sigma \in\left(0, \frac{m \lambda}{b}\right)
$$

and $\sigma u_{n} / \mu_{n} \rightarrow \widehat{u}$ weakly in $H^{1}(\Omega)$ and strongly in $L^{p}\left(\Omega_{0}\right)$ for any $p>1$, where $\widehat{u}$ is a nonnegative function satisfying $\widehat{u}(x)=0$ for $x \in \Omega \backslash \overline{\Omega_{0}}$, and $\left.\widehat{u}\right|_{\Omega_{0}} \in H_{0}^{1}\left(\Omega_{0}\right)$ is a weak solution of the (free boundary) problem

$$
-\Delta u=\left(\lambda u-\frac{b \sigma}{m}\right) \chi_{\{u>0\}}, \quad x \in \Omega_{0}, \quad u(x)=0, \quad x \in \partial \Omega_{0}, \quad\|u\|_{\infty}=1 .
$$


Proof. Let $\left(\mu_{n}, u_{n}\right)$ be a sequence of positive solutions of (3.12) and $\mu_{n} \rightarrow \infty$ as $n \rightarrow$ $\infty$. From a simple comparison argument, we easily deduce $\lambda>b \mu_{n} /\left(1+m\left\|u_{n}\right\|_{\infty}\right)$. Thus

$$
\left\|u_{n}\right\|_{\infty} \geq \frac{b \mu_{n}-\lambda}{m \lambda}
$$

Let $\widehat{u_{n}}=u_{n} /\left\|u_{n}\right\|_{\infty}$. Then $-\Delta \widehat{u_{n}} \leq \lambda \widehat{u_{n}}$. From the proof of Proposition 4.1, we can assume that $\widehat{u_{n}} \rightarrow \widehat{u}$ weakly in $H^{1}(\Omega)$ and strongly in $L^{p}(\Omega)$ for any $p>1$ as $n \rightarrow \infty, \widehat{u} \not \equiv 0$. From Lemma 2.2 of [DD, we also have $\|\widehat{u}\|_{\infty}=1$. From (4.16), $\mu_{n}\left\|u_{n}\right\|_{\infty}^{-1}$ is bounded from above, then from (4.10) with $v_{n}$ replaced by $\mu_{n}$, we obtain

$$
\begin{aligned}
\frac{m}{\left\|u_{n}\right\|_{\infty}} \int_{\Omega}\left|\nabla \widehat{u_{n}}\right|^{2} d x & =\frac{\lambda-b \mu_{n}}{\left\|u_{n}\right\|_{\infty}^{2}} \int_{\Omega} \widehat{u_{n}} d x+\frac{\lambda m}{\left\|u_{n}\right\|_{\infty}} \int_{\Omega}{\widehat{u_{n}}}^{2} d x \\
& -\frac{1}{\left\|u_{n}\right\|_{\infty}} \int_{\Omega} a(x){\widehat{u_{n}}}^{2} d x-m \int_{\Omega} a(x){\widehat{u_{n}}}^{3} d x .
\end{aligned}
$$

Letting $n \rightarrow \infty$, we again obtain (4.11). Hence $\widehat{u}=0$ almost everywhere in $\bar{\Omega} \backslash \overline{\Omega_{0}}$.

From (4.16), we have

$$
\varlimsup_{n \rightarrow \infty} \mu_{n}\left\|u_{n}\right\|_{\infty}^{-1} \leq \frac{m \lambda}{b} .
$$

We claim that $\underline{\lim }_{n \rightarrow \infty} \mu_{n}\left\|u_{n}\right\|_{\infty}^{-1}>0$. Otherwise, there exists a subsequence (still denoted by $\left\{\mu_{n}\right\}$ ) such that $\lim _{n \rightarrow \infty} \mu_{n}\left\|u_{n}\right\|_{\infty}^{-1}=0$. Then from (4.6) and (4.7) with $v_{n}$ replaced by $\mu_{n}$, we find that $\widehat{u}$ satisfies (4.9), which contradicts with $\lambda>$ $\lambda_{1}^{D}\left(\Omega_{0}\right)$. Thus $\underline{\lim }_{n \rightarrow \infty} \mu_{n}\left\|u_{n}\right\|_{\infty}^{-1}>0$. On the other hand, from (4.17) we deduce $\varlimsup_{n \rightarrow \infty} \mu_{n}\left\|u_{n}\right\|_{\infty}^{-1}<m \lambda / b$; for otherwise, we will have

$$
m \int_{\Omega_{0}}|\nabla \widehat{u}|^{2} d x \leq 0
$$

and hence $\widehat{u} \equiv 0$ in $\bar{\Omega}$. Therefore by taking a subsequence, we assume that $\lim _{n \rightarrow \infty} \mu_{n}\left\|u_{n}\right\|_{\infty}^{-1}=\sigma \in(0, m \lambda / b)$.

In $\Omega_{0}, \widehat{u_{n}}$ satisfies

$$
-\Delta \widehat{u_{n}}=\lambda \widehat{u_{n}}-\frac{b \mu_{n} \widehat{u_{n}}}{1+m\left\|u_{n}\right\|_{\infty} \widehat{u_{n}}} .
$$

Since the right side of (4.20) is bounded in $L^{\infty}\left(\Omega_{0}\right)$, we find that $\widehat{u} \in C^{1}(D)$ for any compact subset $D$ of $\Omega_{0}$. We define $\Omega_{0,+}=\left\{x \in \Omega_{0}: \widehat{u}>0\right\}$, and $\Omega_{0,0}=\left\{x \in \Omega_{0}: \widehat{u}=0\right\}$. Then in $\Omega_{0,+}$, from (4.20), and the convergence of $\widehat{u_{n}}$, we obtain

$$
-\Delta \widehat{u}=\lambda \widehat{u}-\frac{b \sigma}{m} .
$$

Clearly $\Delta \widehat{u}=0$ a.e. in $\Omega_{0,0}$. This completes the proof of Proposition 4.4

Remark 4.5. In the one dimensional case, the free boundary problem (4.15) can be completely solved; see the discussion at the end of subsection 4.3 below.

We will also need information on the following perturbed equation:

$$
-\Delta u=\lambda u-[a(x)+\varepsilon] u^{2}-b \mu \frac{u}{1+m u}, x \in \Omega, \quad \partial_{\nu} u=0, x \in \partial \Omega .
$$

With the perturbation, the logistic problem

$$
-\Delta u=\lambda u-[a(x)+\varepsilon] u^{2}, x \in \Omega, \quad \partial_{\nu} u=0, x \in \partial \Omega,
$$


has a unique positive solution $u_{\lambda, \varepsilon}(x)$ for any $\lambda>0$ and $\varepsilon>0$. Moreover, we can see from the following result that the behavior of the global bifurcation branch of the perturbed problem (4.22) is similar to the unperturbed case but with $\lambda<\lambda_{1}^{D}\left(\Omega_{0}\right)$ (see Proposition 3.3).

Proposition 4.6. Suppose that $\varepsilon>0, \lambda>\lambda_{1}^{D}\left(\Omega_{0}\right)$, and $b, m>0$ are fixed. Then $\mu=\lambda / b$ is a bifurcation point for (4.22) such that a global continuum $\Sigma_{\varepsilon}$ of positive solutions of (4.22) emanates from $(\mu, u)=(\lambda / b, 0)$, and

$$
\operatorname{proj}_{\mu} \Sigma_{\varepsilon}=\left(-\infty, \hat{\mu}_{\varepsilon}^{*}\right] \text { or }\left(-\infty, \hat{\mu}_{\varepsilon}^{*}\right)
$$

where $\hat{\mu}_{\varepsilon}^{*}=\sup \{\mu>0:$ (4.22) has a positive solution $\} \geq \lambda / b$. Moreover $\Sigma_{\varepsilon}$ satisfies:

(1) $\operatorname{Near}(\mu, u)=(\lambda / b, 0), \Sigma_{\varepsilon}$ is a curve.

(2) For $\mu \leq 0, \Sigma_{\varepsilon}$ is a smooth curve, and (4.22) has a unique positive solution $\bar{U}_{\mu, \varepsilon}(x)$ for $\mu \leq 0$.

(3) For $\mu \in\left(-\infty, \hat{\mu}_{\varepsilon}^{*}\right)$, (4.22) has a maximal positive solution $\bar{U}_{\mu, \varepsilon}(x)$, and $\bar{U}_{\mu, \varepsilon}$ is strictly decreasing with respect to $\mu$ and $\varepsilon$.

(4) For $\mu \in(-\infty, \lambda / b)$, (4.22) has a minimal positive solution $\underline{U}_{\mu, \varepsilon}(x)$, and $\underline{U}_{\mu, \varepsilon}$ is strictly decreasing with respect to $\mu$ and $\varepsilon$.

(5) If $\hat{\mu}_{\varepsilon}^{*}>\lambda / b$, then (4.22) has a maximal positive solution for $\mu=\hat{\mu}_{\varepsilon}^{*}$ and has at least two positive solutions for $\mu \in\left(\lambda / b, \hat{\mu}_{\varepsilon}^{*}\right)$.

(6) If $\hat{\mu}_{\varepsilon}^{*}>\lambda / b$ and $0<m<m_{0}$, then there exists $\hat{\mu}_{*, \varepsilon} \in(0, \lambda / b)$ such that (4.22) has at least three positive solutions for $\hat{\mu}_{*, \varepsilon}<\mu<\lambda / b$. For $\mu \in\left[\hat{\mu}_{*, \varepsilon}, \lambda / b\right), \underline{U}_{\mu, \varepsilon}<\bar{U}_{\mu, \varepsilon}$, and $\underline{U}_{\mu, \varepsilon} \rightarrow 0$ as $\mu \rightarrow \lambda / b$.

(7) $\hat{\mu}_{\varepsilon}^{*}$ and $\hat{\mu}_{*, \varepsilon}$ are both nonincreasing with respect to $\varepsilon$.

All these solutions mentioned above can be chosen from the unbounded continuum $\Sigma_{\varepsilon}$

Proof. The local bifurcation analysis again is similar to previous ones. The bifurcation diagram of (4.22) cannot be extended to $\mu=\infty$ since, similar to the proof of (3.2), we have, for $\mu>0$,

$$
\lambda=\lambda_{1}^{N}\left((a(x)+\varepsilon) u+\frac{b v}{1+m u}\right)>\lambda_{1}^{N}\left(\frac{b \mu}{1+m\left\|u_{\lambda, \varepsilon}\right\|_{\infty}}\right)=\frac{b \mu}{1+m\left\|u_{\lambda, \varepsilon}\right\|_{\infty}},
$$

where $u_{\lambda, \varepsilon}$ is the unique solution of (4.23). Hence the projection of $\Sigma_{\varepsilon}$ on the $\mu$-axis is bounded from above, and we denote the upper bound by $\hat{\mu}_{\varepsilon}^{*}$. On the other hand, from the maximum principle, any positive solution $u$ of (4.22) satisfies

$$
\|u\|_{\infty} \leq \frac{\lambda}{\varepsilon} \text { if } \mu \geq 0, \quad \text { and }\|u\|_{\infty} \leq \frac{\lambda-b \mu}{\varepsilon} \text { if } \mu<0 .
$$

Thus the projection of $\Sigma_{\varepsilon}$ must cover up to $-\infty$. The proofs for the other parts (except Part 7) are the same as that of Proposition 3.3, thus we omit them here.

For Part 7 , to see that $\hat{\mu}_{\varepsilon}^{*}$ is nonincreasing with respect to $\varepsilon$, we note that if $\varepsilon_{1}<\varepsilon_{2}$ and $\hat{\mu}_{\varepsilon_{2}}^{*}=\lambda / b$, then $\hat{\mu}_{\varepsilon_{1}}^{*} \geq \hat{\mu}_{\varepsilon_{2}}^{*}$ follows trivially from the fact that $\hat{\mu}_{\varepsilon}^{*} \geq \lambda / b$; if $\hat{\mu}_{\varepsilon_{2}}^{*}>\lambda / b$, then (4.22) with $\epsilon=\varepsilon_{2}$ and $\mu=\hat{\mu}_{\varepsilon_{2}}^{*}$ has a maximal solution $\bar{U}$, which is a subsolution for (4.22) with $\varepsilon=\varepsilon_{1}$ and $\mu=\hat{\mu}_{\varepsilon_{2}}^{*}+\delta$ if $\delta$ is sufficiently small. Since $u_{\lambda, \varepsilon_{1}}$ is a supersolution above $\bar{U}$, 4.22) has a positive solution with $\epsilon=\varepsilon_{1}$ and $\mu=\hat{\mu}_{\varepsilon_{2}}^{*}+\delta$. Therefore, $\hat{\mu}_{\varepsilon_{1}}^{*} \geq \hat{\mu}_{\varepsilon_{2}}^{*}+\delta$. The proof for $\hat{\mu}_{*, \varepsilon}$ is similar. 
We will show in the following that as $\varepsilon \rightarrow 0, \hat{\mu}_{\varepsilon}^{*} \rightarrow \infty$. This helps us to understand how $\Sigma_{\varepsilon}$ in Proposition 4.6 is related to $\Sigma_{0}$ in Proposition 4.3, Roughly speaking, as $\varepsilon \rightarrow 0$, the part of $\Sigma_{\varepsilon}$ containing the maximal solutions blows up, and the remaining part converges to $\Sigma_{0}$.

Proposition 4.7. Suppose that $\lambda>\lambda_{1}^{D}\left(\Omega_{0}\right)$. Then $\hat{\mu}_{\varepsilon}^{*} \rightarrow \infty$ as $\epsilon \rightarrow 0$, and for any given $\mu, \lim _{\varepsilon \rightarrow 0}\left\|\bar{U}_{\mu, \varepsilon}\right\|_{\infty} \rightarrow \infty$. Moreover, let $\left(\mu_{n}, \varepsilon_{n}, u_{n}\right)$ be a sequence of positive solutions of (4.22) with $\mu_{n} \rightarrow \mu_{0}$ and $\lim _{n \rightarrow \infty}\left\|u_{n}\right\|_{\infty}=\infty$. Then, subject to a subsequence,

$$
\begin{aligned}
& u_{n} \rightarrow \infty \text { uniformly on } \overline{\Omega_{0}} \text { as } \varepsilon_{n} \rightarrow 0, \\
& \epsilon_{n} u_{n} \rightarrow \phi_{\lambda} \text { uniformly on } \overline{\Omega_{0}} \text { as } \varepsilon_{n} \rightarrow 0, \\
& u_{n} \rightarrow U_{\mu_{0}}^{\lambda} \text { uniformly on any compact subset of } \bar{\Omega} \backslash \overline{\Omega_{0}} \text { as } \varepsilon_{n} \rightarrow 0,
\end{aligned}
$$

where $\phi_{\lambda}$ is the unique positive solution of

$$
-\Delta u=\lambda u-u^{2}, x \in \Omega_{0} ; u=0, x \in \partial \Omega_{0},
$$

and $U_{\mu_{0}}^{\lambda}$ is a positive solution of the boundary blow-up problem

$-\Delta u=\lambda u-a(x) u^{2}-b \mu_{0} \frac{u}{1+m u}, x \in \Omega \backslash \bar{\Omega}_{0} ; \partial_{\nu} u=0, x \in \partial \Omega ; u=\infty, x \in \partial \Omega_{0}$.

Proof. By the anti-maximum principle $[\mathrm{CP}$, the equation

$$
-\Delta Z=\lambda_{a} Z-\frac{b}{m}, x \in \Omega_{0}, u=0, x \in \partial \Omega_{0},
$$

has a unique positive solution $Z$ for any fixed $\lambda_{a} \in\left(\lambda_{1}^{D}\left(\Omega_{0}\right), \lambda_{1}^{D}\left(\Omega_{0}\right)+\delta\right)$ with a small $\delta>0$. Since $\lambda>\lambda_{1}^{D}\left(\Omega_{0}\right)$, we can choose $\lambda_{a}$ so that the anti-maximum principle holds and $\lambda-\lambda_{a}=\eta>0$. Then for any given $\mu>0, \sigma>\mu$ and $x \in \Omega_{0}$, we have

$$
\begin{aligned}
& \Delta(\sigma Z)+\lambda(\sigma Z)-\frac{b \mu \sigma Z}{1+m \sigma Z}-[a(x)+\varepsilon](\sigma Z)^{2} \\
= & \left(\lambda-\lambda_{a}-\varepsilon \sigma Z\right) \sigma Z+b \sigma\left(\frac{1}{m}-\frac{\mu Z}{1+m \sigma Z}\right) \geq(\eta-\varepsilon \sigma Z) \sigma Z .
\end{aligned}
$$

For any compact subset $P \subset \subset \Omega_{0}, Z_{m}=\min _{x \in P} Z(x)>0$. For any $M>0$, we can choose $\sigma>0$ such that $\sigma Z_{m} \geq M$; thus $\sigma Z(x) \geq M$ on $P$. On the other hand if we choose $\varepsilon<\eta /\left(2 \sigma Z_{m}\right)$, then $\eta-\varepsilon \sigma Z \geq 0$ for $x \in \Omega_{0}$, and $\sigma Z$ extended to be zero outside $\Omega_{0}$ is a weak subsolution of (4.22) (see [BL]). Clearly it is also a weak subsolution of (4.23). It follows that $u_{\lambda, \epsilon} \geq \sigma Z$. Moreover, $u_{\lambda, \epsilon}$ is a supersolution of (4.22). Therefore (4.22) has a positive solution with this $\mu$ and $\bar{U}_{\mu, \varepsilon} \geq \sigma Z \geq M$ on $P$. This implies that $\hat{\mu}_{\varepsilon}^{*} \rightarrow \infty$ as $\varepsilon \rightarrow 0$ and $\left\|\bar{U}_{\mu, \varepsilon}\right\|_{\infty} \rightarrow \infty$ as $\epsilon \rightarrow 0$ for any $\mu>0$. By the monotonicity of $\bar{U}_{\mu, \varepsilon}$ with respect to $\mu$, the above limit also holds for $\mu \leq 0$. This proves the first part of the proposition. We remark that the anti-maximum principle was used in [OSS] to construct a subsolution in a similar way.

To prove the conclusions on $u_{n}$, similar to the proof of Proposition 4.1, we define $\widehat{u_{n}}=u_{n} /\left\|u_{n}\right\|_{\infty}$. Then again $-\Delta \widehat{u_{n}} \leq \lambda \widehat{u_{n}}$, and we can assume that $\widehat{u_{n}} \rightarrow \widehat{u}$ weakly in $H^{1}(\Omega)$ and strongly in $L^{p}(\Omega)$ for any $p>1$ as $n \rightarrow \infty, \widehat{u} \not \equiv 0$, and $\|\widehat{u}\|_{\infty}=1$. From (4.25), $\varepsilon_{n}\left\|u_{n}\right\|_{\infty} \leq \lambda$. Arguing the same way as in the proof of Proposition 4.4, we obtain that $\widehat{u}=0$ almost everywhere in $\bar{\Omega} \backslash \overline{\Omega_{0}}$. 
Since $\varepsilon_{n}\left\|u_{n}\right\|_{\infty} \leq \lambda$, we can assume (subject to a subsequence) $\lim _{n \rightarrow \infty} \varepsilon_{n}\left\|u_{n}\right\|_{\infty}$ $=L \leq \lambda$. We claim that $L>0$. Suppose this is not true; then $\lim _{n \rightarrow \infty} \varepsilon_{n}\left\|u_{n}\right\|_{\infty}=$ 0 along a subsequence. For any $\phi \in H_{0}^{1}\left(\Omega_{0}\right)$, we have the obvious variations of (4.6) and (4.7) with $v_{n}$ replaced by $\mu_{n}$. If $L=0$, then we can similarly obtain (4.8) and (4.9) and we again get $\widehat{u} \equiv 0$, which contradicts $\|\widehat{u}\|_{\infty}=1$. Thus $L>0$. We can now easily see that $\left.\widehat{u}\right|_{\Omega_{0}}$ is the unique positive solution of

$$
-\Delta u=\lambda u-L u^{2}, x \in \Omega_{0}, \quad u(x)=0, x \in \partial \Omega_{0} .
$$

Hence $\varepsilon_{n} u_{n}=\left(\varepsilon_{n}\left\|u_{n}\right\|_{\infty}\right) \widehat{u_{n}} \rightarrow L \widehat{u}=\phi_{\lambda}$ in $L^{p}\left(\Omega_{0}\right)$ for any $p>1$. Moreover, we can use standard interior estimates of elliptic operators to conclude that this convergence is uniform for $x$ in any compact subset of $\Omega_{0}$.

It is known (see D5, Theorem 2.7) that $\varepsilon u_{\lambda, \epsilon} \rightarrow \phi_{\lambda}$ uniformly in $\bar{\Omega}_{0}$. By a simple comparison argument we find $u_{n} \leq u_{\lambda, \varepsilon_{n}}$ and therefore $0 \leq \varepsilon_{n} u_{n} \leq \varepsilon_{n} u_{\lambda, \varepsilon_{n}}$. It follows that for all large $n, \varepsilon_{n} u_{n}$ is uniformly close to 0 near $\partial \Omega_{0}$. Together with our earlier conclusion, we find that $\varepsilon_{n} u_{n} \rightarrow \phi_{\lambda}$ uniformly on $\bar{\Omega}_{0}$.

Now for large enough $n, u_{n}(x) \geq \varepsilon_{n}^{-1} \varphi_{\lambda}(x)-1 /(2 m)$ for $x \in \Omega_{0}$. It follows that

$$
\frac{b \mu_{n}}{1+m u_{n}} \leq \Phi_{n}:=\frac{b\left|\mu_{0}\right|+1}{1 / 2+m \varepsilon_{n}^{-1} \phi_{\lambda}} \rightarrow 0
$$

in $L^{p}\left(\Omega_{0}\right)$ for any $p>1$.

Let $\psi_{1}$ be the positive eigenfunction of

$$
-\Delta \psi=\lambda_{1}^{D}\left(\Omega_{0}\right) \psi, x \in \Omega_{0}, \quad \psi(x)=0, x \in \partial \Omega_{0},
$$

such that $\left\|\psi_{1}\right\|_{L^{2}\left(\Omega_{0}\right)}=1$. Then for all large $n$,

$$
\begin{aligned}
\lambda_{1}^{D}\left(\Omega_{0}\right) & \leq \lambda_{1}^{D}\left(\Phi_{n}, \Omega_{0}\right)=\min _{\psi \in H_{0}^{1}\left(\Omega_{0}\right),\|\psi\|_{L^{2}\left(\Omega_{0}\right)}=1} \int_{\Omega_{0}}\left[|\nabla \psi|^{2}+\Phi_{n} \psi^{2}\right] d x \\
& \leq \int_{\Omega_{0}}\left[\left|\nabla \psi_{1}\right|^{2}+\Phi_{n} \psi_{1}^{2}\right] d x=\lambda_{1}^{D}\left(\Omega_{0}\right)+\int_{\Omega_{0}} \Phi_{n} \psi_{1}^{2} d x \\
& \leq \lambda_{1}^{D}\left(\Omega_{0}\right)+\left\|\Phi_{n}\right\|_{p}\left\|\psi_{1}\right\|_{q}^{2} \rightarrow \lambda_{1}^{D}\left(\Omega_{0}\right),
\end{aligned}
$$

where $q>2$ is such that $H_{0}^{1}\left(\Omega_{0}\right)$ imbeds continuously into $L^{q}\left(\Omega_{0}\right)$ and $p=q /(q-1)$. Therefore

$$
\lambda_{1}^{D}\left(\Omega_{0}\right)=\lim _{n \rightarrow \infty} \lambda_{1}^{D}\left(\Phi_{n}, \Omega_{0}\right) .
$$

It follows that, for all large $n, \lambda>\lambda_{1}^{D}\left(\Phi_{n}, \Omega_{0}\right)$. Let $V_{n}$ be the unique solution of

$$
-\Delta u=\left(\lambda-\frac{b\left|\mu_{0}\right|+1}{1+m U}\right) u-\left[a(x)+\varepsilon_{n}\right] u^{2}, x \in \Omega, \quad \partial_{\nu} u=0, x \in \partial \Omega,
$$

where $U=\varepsilon_{n_{1}}^{-1} \phi_{\lambda}-1 /(2 m)$ in $\Omega_{0}$ and is extended to be $-1 /(2 m)$ outside $\Omega_{0}$, and $n_{1}$ is large but fixed. Then for $n \geq n_{1}, u_{n} \geq V_{n}$ from the comparison principle and the uniqueness of a positive solution of (4.34). Now from Theorem 2.1 of [D2, we find

$$
V_{n} \rightarrow \infty \text { uniformly on } \overline{\Omega_{0}} \text { as } \varepsilon \rightarrow 0 .
$$

It follows from $u_{n} \geq V_{n}$ that $u_{n} \rightarrow \infty$ uniformly on $\bar{\Omega}_{0}$. The last conclusion in (4.26) follows from a standard compactness argument. 
4.3. Dynamical behavior of the scalar equations. In this subsection, we will first consider the dynamics of the perturbed scalar equation:

$$
\begin{cases}u_{t}-\Delta u=\lambda u-[a(x)+\varepsilon] u^{2}-b \mu \frac{u}{1+m u}, & x \in \Omega, t>0, \\ \partial_{\nu} u=0, & x \in \partial \Omega, t>0, \\ u(x, 0)=u_{0}(x) \geq 0, & x \in \Omega .\end{cases}
$$

Then we will use the results of (4.36) to study the dynamics of the unperturbed scalar equation (3.29), and finally we will consider the dynamics of the full system (2.1) in the next subsection.

Theorem 4.8. Suppose that $\varepsilon>0, \lambda>\lambda_{1}^{D}\left(\Omega_{0}\right)$, and $b, m>0$ are fixed. Let $\hat{\mu}_{\varepsilon}^{*}$ and $\hat{\mu}_{\varepsilon, *}$ be defined as in Proposition 4.6. Then all solutions $u(x, t)$ of (4.36) are globally bounded, and the following hold true:

(1) If $\mu \leq 0$, then $\bar{U}_{\mu, \varepsilon}$ is globally asymptotically stable.

(2) If $\mu>\hat{\mu}_{\varepsilon}^{*}$, then 0 is globally asymptotically stable.

(3) If $0<\mu<\lambda / b$, then for any nontrivial $u_{0}, \underline{\lim }_{t \rightarrow \infty} u(x, t) \geq \underline{U}_{\mu, \varepsilon}(x)$.

(4) If $0<\mu \leq \hat{\mu}_{\varepsilon}^{*}$, then for any nontrivial $u_{0}, \overline{\lim }_{t \rightarrow \infty} u(x, t) \leq \bar{U}_{\mu, \varepsilon}(x)$, and if $u_{0}(x) \geq \bar{U}_{\mu, \varepsilon}(x)$, then $\lim _{t \rightarrow \infty} u(x, t)=\bar{U}_{\mu, \varepsilon}(x)$; if $u_{0}(x) \geq \bar{U}_{\hat{\mu}_{\varepsilon}^{*}, \varepsilon}(x)$, then

$$
V_{\mu, 3}(x) \leq \varliminf_{t \rightarrow \infty} u(x, t) \leq \varlimsup_{t \rightarrow \infty} u(x, t) \leq \bar{U}_{\mu, \varepsilon}(x),
$$

where $V_{\mu, 3}(x)$ is the unique positive solution of (3.31) with $U=\bar{U}_{\hat{\mu}_{\varepsilon}^{*}, \varepsilon}$.

(5) If $\hat{\mu}_{*, \varepsilon}<\lambda / b, \hat{\mu}_{*, \varepsilon}<\mu<\lambda / b$, and $u_{0}(x) \leq \underline{U}_{\mu, \varepsilon}(x)$, then $\lim _{t \rightarrow \infty} u(x, t)=$ $\underline{U}_{\mu, \varepsilon}(x)$, and if $u_{0}(x) \leq \underline{U}_{\hat{\mu}_{*, \varepsilon}, \varepsilon}(x)$, then

$$
\underline{U}_{\mu, \varepsilon}(x) \leq \underline{\lim }_{t \rightarrow \infty} u(x, t) \leq \varlimsup_{\lim } \rightarrow \infty u(x, t) \leq V_{\mu, 4}(x),
$$

where $V_{\mu, 4}$ is the unique positive solution of (3.31) with $U=\underline{U}_{\hat{\mu}_{*, \varepsilon}, \varepsilon}$.

(6) If $\hat{\mu}_{*, \varepsilon}<\lambda / b$, then there exists $\hat{\mu}_{\varepsilon}^{\sharp} \in\left(\lambda / b, \hat{\mu}_{\varepsilon}^{*}\right)$ such that $\lambda=$ $\lambda_{1}^{N}\left(b \hat{\mu}_{\varepsilon}^{\sharp} /\left(1+m \underline{U}_{\hat{\mu}_{*, \varepsilon}, \varepsilon}\right)\right)$, and for $\lambda / b \leq \mu<\hat{\mu}_{\varepsilon}^{\sharp}$, if $u_{0}(x) \leq \underline{U}_{\hat{\mu}_{*, \varepsilon}, \varepsilon}(x)$, then

$$
0 \leq \underline{\lim }_{t \rightarrow \infty} u(x, t) \leq \varlimsup_{t \rightarrow \infty} u(x, t) \leq V_{\mu, 4}(x) .
$$

For $\mu \geq \hat{\mu}_{\varepsilon}^{\sharp}$, if $u_{0}(x) \leq \underline{U}_{\hat{\mu}_{* \varepsilon}, \varepsilon}(x)$, then $\lim _{t \rightarrow \infty} u(x, t)=0$.

The proof of Theorem 4.8 is exactly the same as that of Theorem 3.6 thus we omit it. Now we consider the dynamics of the unperturbed scalar equation (3.29).

Theorem 4.9. Suppose that $\lambda>\lambda_{1}^{D}\left(\Omega_{0}\right)$ and that $b, m>0$ are fixed. Let $u(x, t)$ be a solution of (3.29). Then the following hold:

(1) If $\mu<\mu_{*}^{0}$, then for any $u_{0}, u(x, t) \rightarrow \infty$ uniformly for $x \in \overline{\Omega_{0}}$, and $\overline{\lim }_{t \rightarrow \infty} u(x, t) \leq \bar{U}^{\lambda}(x)$ for $x \in \bar{\Omega} \backslash \overline{\Omega_{0}}$, where $\bar{U}^{\lambda}$ is the maximum solution of (2.6).

(2) If $\mu_{*}^{0}<\lambda / b$, and $u_{0}(x) \leq \underline{U}_{\mu_{*}^{0}}(x)$, then for $\mu \in\left[\mu_{*}^{0}, \lambda / b\right)$,

$$
\underline{U}_{\mu}(x) \leq \underline{\lim }_{t \rightarrow \infty} u(x, t) \leq \varlimsup_{t \rightarrow \infty} u(x, t) \leq V_{\mu, 5}(x),
$$

where $V_{\mu, 5}$ is the solution of (3.31) with $U=\underline{U}_{\mu_{*}^{0}}$, and for $\mu \geq \lambda / b$,

$$
0 \leq \underline{\lim }_{t \rightarrow \infty} u(x, t) \leq \varlimsup_{t \rightarrow \infty} u(x, t) \leq \max \left\{V_{\mu, 5}(x), 0\right\} .
$$


(3) For any given $\widehat{\mu}>0$ we define

$$
C_{\widehat{\mu}}=\sup \left\{\|u\|_{\infty}: u \text { is a positive solution of (3.12) with } \mu \leq \widehat{\mu}\right\}
$$

(the supremum exists from Proposition 4.1). Let $\mu_{0}>\widehat{\mu}$ be such that

$$
C_{\widehat{\mu}}<\left(b \mu_{0}-\lambda\right) /(m \lambda)
$$

and let $U_{\mu_{0}}(x)$ be a positive solution of (3.12) with $\mu=\mu_{0}$. Then for $\mu \leq \widehat{\mu}$ and $u_{0}(x) \geq U_{\mu_{0}}(x)$, the solution $u(x, t) \rightarrow \infty$ uniformly for $x \in \overline{\Omega_{0}}$, and $\varlimsup_{t \rightarrow \infty} u(x, t) \leq \bar{U}^{\lambda}(x)$ for $x \in \bar{\Omega} \backslash \overline{\Omega_{0}}$.

(4) Let $U_{\mu_{0}}(x)$ be a positive solution of (3.12) with $\mu=\mu_{0}>0$. Define $\widetilde{\mu}^{*}$ to be the unique positive number such that $\lambda=\lambda_{1}^{N}\left(b \widetilde{\mu}^{*} /\left(1+m U_{\mu_{0}}\right)\right)$. Then for $\mu>\widetilde{\mu}^{*}$ and $u_{0}(x) \leq U_{\mu_{0}}(x), \lim _{t \rightarrow \infty} u(x, t)=0$ uniformly for $x \in \bar{\Omega}$.

Proof. First we assume that $\mu<\mu_{*}^{0}$. Let $u_{\varepsilon}(x, t)$ be the solution of (4.36) with the same initial condition $u_{0}(x)$. Then $u(x, t) \geq u_{\varepsilon}(x, t)$ for any $\varepsilon>0$ from the comparison principle. From Theorem 4.8, $u_{\varepsilon}(x, t)$ is globally bounded, and $\underline{\lim }_{t \rightarrow \infty} u_{\varepsilon}(x, t) \geq \underline{U}_{\mu, \varepsilon}(x)$. We claim that $\left\|\underline{U}_{\mu, \varepsilon}\right\|_{\infty} \rightarrow \infty$ as $\varepsilon \rightarrow 0$. If not, due to the monotonicity of $\underline{U}_{\mu, \varepsilon}$ with respect to $\varepsilon$, it converges to a positive solution of (3.12) as $\varepsilon \rightarrow 0$, which contradicts $\mu<\mu_{*}^{0}$. Using Proposition 4.7, $\underline{U}_{\mu, \varepsilon}$ satisfies (4.26) as $\varepsilon \rightarrow 0$, which implies $u(x, t) \geq u_{\varepsilon}(x, t) \rightarrow \infty$ uniformly for $x \in \overline{\Omega_{0}}$. On the other hand, comparing $u(x, t)$ to the solution of (2.4) with the same initial condition, we obtain $\overline{\lim }_{t \rightarrow \infty} u(x, t) \leq \bar{U}^{\lambda}(x)$ for $x \in \bar{\Omega} \backslash \overline{\Omega_{0}}$. This completes the proof of Part 1, and the proof of Part 2 is similar to that of Theorem 3.6.

For Part 3, when $\mu \leq \widehat{\mu},\|u\|_{\infty} \leq C_{\widehat{\mu}}<\left(b \mu_{0}-\lambda\right) /(m \lambda)$ for any positive solution $u$ of (3.12), but by (4.16), $\left\|U_{\mu_{0}}\right\|_{\infty} \geq\left(b \mu_{0}-\lambda\right) /(m \lambda)$. Hence for such a $\mu$, there is no steady state solution $u$ such that $u \geq U_{\mu_{0}}$. On the other hand, since $U_{\mu_{0}}$ is a steady state and $\mu_{0}>\widehat{\mu} \geq \mu$, we find that $U_{\mu_{0}}$ is a subsolution of (3.29); thus the solution $u_{1}(x, t)$ of (3.29) with initial value $U_{\mu_{0}}$ is strictly increasing in $t$. Since $u_{0} \geq U_{\mu_{0}}$, we have $u(x, t) \geq u_{1}(x, t)$; thus we only need to show that $u_{1}$ blows up as $t \rightarrow \infty$. To prove that, we compare $u_{1}(x, t)$ with $u_{1, \varepsilon}(x, t)$, the solution of (4.36) with initial value $U_{\mu_{0}}$. Since $U_{\mu_{0}}$ is also a subsolution of (4.36), $u_{1, \varepsilon}(x, t)$ increases in $t$, and $w_{\varepsilon}(x):=\varliminf_{t \rightarrow \infty} u_{1, \varepsilon}(x, t)$ is a positive steady state solution and $w_{\varepsilon} \geq U_{\mu_{0}}$. We must have $\left\|w_{\varepsilon}\right\|_{\infty} \rightarrow \infty$ as $\varepsilon \rightarrow 0$; for otherwise along a subsequence, $w_{\varepsilon}$ converges to a positive solution $w$ of (3.12) and $w \geq U_{\mu_{0}}$, a contradiction to our earlier observation. Therefore $\left\|w_{\varepsilon}\right\|_{\infty} \rightarrow \infty$ and by Proposition 4.7, we deduce $w_{\varepsilon} \rightarrow \infty$ uniformly in $\bar{\Omega}_{0}$, which implies $\varliminf_{t \rightarrow \infty} u(x, t)=\infty$ uniformly on $\bar{\Omega}_{0}$. The last conclusion in Part 3 can be proved in the same way as in Part 1.

For Part 4, we compare $u(x, t)$ with $u_{2}(x, t)$, the solution of (3.34) with $U=U_{\mu_{0}}$ and the same initial value. The function $\mu \mapsto \lambda_{1}^{N}\left(b \mu /\left(1+m U_{\mu_{0}}\right)\right)$ is increasing in $\mu$. So when $\mu>\widetilde{\mu}^{*}, \lambda<\lambda_{1}^{N}\left(b \mu /\left(1+m U_{\mu_{0}}\right)\right)$, and the zero steady state solution of (3.34) is globally asymptotically stable; thus $u_{2}(x, t) \rightarrow 0$ as $t \rightarrow \infty$. On the other hand, from

$$
\lambda=\lambda_{1}^{N}\left(a U_{\mu_{0}}+\frac{b \mu_{0}}{1+m U_{\mu_{0}}}\right)>\lambda_{1}^{N}\left(\frac{b \mu_{0}}{1+m U_{\mu_{0}}}\right)
$$

we deduce $\widetilde{\mu}^{*}>\mu_{0}$, and hence $U_{\mu_{0}}$ is a supersolution of (3.29). It follows that $0 \leq u(x, t) \leq u_{2}(x, t) \rightarrow 0$ as $t \rightarrow \infty$ uniformly for $x \in \bar{\Omega}$.

In the next proposition, we use a different subsolution to show the blow-up of $u(x, t)$. 
Proposition 4.10. Suppose that $\lambda>\lambda_{1}^{D}\left(\Omega_{0}\right)$ and that $b, m>0$ are fixed. Suppose that $U_{0}(x)$ is a nontrivial solution of

$$
-\Delta u=\left(\lambda_{a} u-\frac{b}{m}\right) \chi_{\{u>0\}}, \quad x \in \Omega_{0}, u(x)=0, \quad x \in \partial \Omega_{0},
$$

where $\lambda_{1}^{D}\left(\Omega_{0}\right)<\lambda_{a}<\lambda$. We extend $U_{0}$ by $U_{0}(x) \equiv 0$ for $x \in \bar{\Omega} \backslash \overline{\Omega_{0}}$. Then there exists $\sigma>0$ such that for $u_{0}(x) \geq \sigma U_{0}(x)$, the solution of (3.29) satisfies $u(x, t) \rightarrow \infty$ uniformly for $x \in \overline{\Omega_{0}}$, and $\overline{\lim }_{t \rightarrow \infty} u(x, t) \leq \bar{U}^{\lambda}(x)$ for $x \in \bar{\Omega} \backslash \overline{\Omega_{0}}$.

Proof. Define $\Omega_{0,+}=\left\{x \in \Omega_{0}: U_{0}(x)>0\right\}$ and $\Omega_{0,0}=\left\{x \in \Omega_{0}: U_{0}(x)=0\right\}$. Then for $x \in \Omega_{0,+}$ and $\sigma>\mu$,

$$
-\Delta\left(\sigma U_{0}\right)=\lambda_{a} \sigma U_{0}-\frac{b \sigma}{m} \leq \lambda \sigma U_{0}-\frac{b \sigma \mu U_{0}}{1+m \sigma U_{0}} .
$$

Thus $\sigma U_{0}$ is a subsolution of (3.12) in $\Omega_{0,+}$. On the other hand $U_{0}=0$ is also a subsolution of (3.12) in $\Omega_{0,0} \cup\left(\bar{\Omega} \backslash \overline{\Omega_{0}}\right)$. Therefore $\sigma U_{0}$ is a subsolution of (3.12) in $\Omega_{0}$. Let $C_{\mu}$ be the constant defined in (4.42). We may assume that $\sigma\left\|U_{0}\right\|_{\infty}>C_{\mu}$ also holds. Then similar to the proof of Theorem 4.9, we can deduce $\underline{\lim }_{t \rightarrow \infty} u(x, t) \geq \underline{\lim }_{t \rightarrow \infty} u_{\varepsilon}(x, t)=w_{\varepsilon}(x) \geq \sigma U_{0}(x)$. But since $\sigma\left\|U_{0}\right\|_{\infty}>C_{\mu}$, we must have $\left\|w_{\varepsilon}\right\|_{\infty} \rightarrow \infty$ as $\varepsilon \rightarrow 0$, and the remaining part of the proof is the same as that of Theorem 4.9 .

To conclude this subsection, we give a complete solution to the free boundary problem (4.43) when the space dimension is one, that is, when $\Omega_{0}$ is a finite interval. This sheds lights on the applications of Proposition 4.10 to the original predatorprey system, especially concerning the problem of biological invasion (see subsection 4.5 below).

For definiteness, we assume that $\Omega_{0}=(-\pi, \pi)$. Moreover, we replace $\lambda_{a}$ by $\lambda$ for convenience of notation. Then $\lambda_{i}^{D}\left(\Omega_{0}\right)=(i / 2)^{2}$, and if $u$ is a solution and $[\alpha, \beta]$ is a component of its supporting set, then $v=u-b /(\lambda m)$ solves $-v^{\prime \prime}=\lambda v$ over $(\alpha, \beta)$. It follows that $v=\sigma \cos (s+\sqrt{\lambda} x)$ for some $\sigma$ and $s$. Thus

$$
u=\sigma \cos (s+\sqrt{\lambda} x)+b /(\lambda m) .
$$

From this expression and the assumption that $[\alpha, \beta]$ is a component of the supporting set of $u$, we see that $u(x)$ is symmetric in $[\alpha, \beta]$ about $x=(\alpha+\beta) / 2$, and it is increasing over $(\alpha,(\alpha+\beta) / 2)$.

If $[\alpha, \beta]=[-\pi, \pi]$, then from the boundary conditions and the symmetry, we necessarily have $s=0$ and $1 / 4<\lambda \leq 1$, and $\sigma>0$ is uniquely determined by

$$
\sigma \cos (\sqrt{\lambda} \pi)+b /(\lambda m)=0 .
$$

Thus there is a unique solution when $\lambda \in(1 / 4,1]$.

The case $[\alpha, \beta] \neq[-\pi, \pi]$ is more complicated. In this situation, $u^{\prime}$ and $u$ both vanish at an endpoint of the interval $[\alpha, \beta]$, and by symmetry, they vanish at both endpoints, namely $u(\alpha)=u(\beta)=u^{\prime}(\alpha)=u^{\prime}(\beta)=0$. In view of the monotonicity property of $u$ and the formula for $u$ given above, we must have

$$
s+\sqrt{\lambda} \alpha=-\pi+2 k \pi, s+\sqrt{\lambda} \beta=\pi+2 k \pi
$$

for some integer $k$, and hence $\lambda=[2 \pi /(\beta-\alpha)]^{2}>1$. Now from $u(\alpha)=u(\beta)=0$ we find $\sigma \cos (\pi)+b /(\lambda m)=0$, that is,

$$
\sigma=b /(\lambda m) .
$$


Therefore, over each component of its supporting set, $u(x)$ is a shift of

$$
\phi_{\lambda}(x)=\frac{b}{\lambda m}[\cos (\sqrt{\lambda} x)+1], x \in[-\pi / \sqrt{\lambda}, \pi / \sqrt{\lambda}] .
$$

This implies that if $\phi_{\lambda}$ is extended to be zero outside its supporting set $[-\pi / \sqrt{\lambda}$, $\pi / \sqrt{\lambda}]$, then any solution of (4.43) with $\lambda>1$ can be expressed as

$$
u(x)=\sum_{i=1}^{k} \phi_{\lambda}\left(x_{i}+x\right), 1 \leq k \leq[\sqrt{\lambda}],
$$

for some $\left\{x_{i}\right\}_{i=1}^{k}$ satisfying

$$
2\left|x_{i}-\pi\right|, 2\left|x_{i}+\pi\right|,\left|x_{i}-x_{j}\right| \geq \frac{2 \pi}{\sqrt{\lambda}}
$$

where $[\sqrt{\lambda}]$ denotes the largest positive integer no greater than $\sqrt{\lambda}$. Conversely, it is clear that any function $u(x)$ given by the above formula is a solution of (4.43) with $\lambda>1$.

For later discussions in subsection 4.5, let us note that when $\lambda$ is large, some solutions of (4.43) have very narrow supporting sets; for example, there is always a solution with supporting set $\left(x_{0}-\pi / \sqrt{\lambda}, x_{0}+\pi / \sqrt{\lambda}\right) \subset(-\pi, \pi)$.

From the above discussion, we find that, when $\Omega_{0}=(-\pi, \pi)$ and $1 / 4<\lambda \leq 1$, for problem (4.15) to have a solution, necessarily

$$
\sigma=\frac{\lambda m}{b}\left(1-\frac{1}{\cos (\sqrt{\lambda} \pi)}\right)^{-1}
$$

and in this case, there is a unique solution:

$$
u(x)=\frac{b \sigma}{\lambda m}\left(1-\frac{\cos (\sqrt{\lambda} x)}{\cos (\sqrt{\lambda} \pi)}\right) .
$$

If $\lambda>1$, then necessarily $\sigma=(\lambda m) /(2 b)$ and the solutions are given by

$$
u(x)=\sum_{i=1}^{k} \frac{\lambda m}{2 b} \phi_{\lambda}\left(x_{i}+x\right), 1 \leq k \leq[\sqrt{\lambda}]
$$

with $x_{i}$ satisfying (4.45).

4.4. Dynamical behavior of the predator-prey system. Finally we consider the dynamics of (2.1) under the assumption $\lambda>\lambda_{1}^{D}\left(\Omega_{0}\right)$.

Theorem 4.11. Suppose that $\lambda>\lambda_{1}^{D}\left(\Omega_{0}\right)$ and that $b, c, m>0$ are fixed. Let $(u(x, t), v(x, t))$ be the solution of (2.1). Then for any nonnegative $\left(u_{0}, v_{0}\right)$ with $u_{0}, v_{0} \not \equiv 0$ and any $\mu \in \mathbf{R}, v(x, t)$ satisfies

$$
\max \{\mu, 0\} \leq \underline{\lim }_{t \rightarrow \infty} v(x, t) \leq \varlimsup_{t \rightarrow \infty} v(x, t) \leq \max \left\{\mu+\frac{c}{m}, 0\right\},
$$


and the asymptotic behavior of $u(x, t)$ is as follows:

(1) If $\mu<\mu_{*}^{0}-c / m$, then $u(x, t)$ satisfies

$$
\begin{aligned}
& \lim _{t \rightarrow \infty} u(x, t)=\infty \text { uniformly on } \overline{\Omega_{0}}, \\
& \bar{U}^{\lambda}(x) \geq \overline{\lim }_{t \rightarrow \infty} u(x, t) \geq \underline{\lim }_{t \rightarrow \infty} u(x, t) \geq 0, x \in \bar{\Omega} \backslash \overline{\Omega_{0}} .
\end{aligned}
$$

If in addition, $\mu<-c / m$, then $\lim _{t \rightarrow \infty} v(x, t)=0$ uniformly on $\bar{\Omega}$.

(2) If $\mu_{*}^{0}+c / m<\lambda / b$, and $u_{0}(x) \leq \underline{U}_{\mu_{*}^{0}}(x)$, then for $\mu \in\left[\mu_{*}^{0}+c / m, \lambda / b\right]$,

$$
\underline{U}_{\mu+c / m}(x) \leq \underline{\lim }_{t \rightarrow \infty} u(x, t) \leq \varlimsup_{t \rightarrow \infty} u(x, t) \leq V_{\mu, 5}(x),
$$

where $V_{\mu, 5}$ is the solution of (3.31) with $U=\underline{U}_{\mu_{*}^{0}}$, and for $\mu>\lambda / b$,

$$
0 \leq \varliminf_{t \rightarrow \infty} u(x, t) \leq \varlimsup_{t \rightarrow \infty} u(x, t) \leq \max \left\{V_{\mu, 5}(x), 0\right\} .
$$

(3) For any given $\widehat{\mu}>0$, let $C_{\widehat{\mu}}, \mu_{0}$ and $U_{\mu_{0}}$ be as in Part 3 of Theorem 4.9. Then for $\mu<\widehat{\mu}-c / m$ and $u_{0}(x) \geq U_{\mu_{0}}(x)$, (4.47) holds.

(4) Let $U_{\mu_{0}}(x)$ be a positive solution of (3.12) with $\mu=\mu_{0}>0$, and let $\widetilde{\mu}^{*}$ be defined as in Theorem 4.9. Then for $\mu>\widetilde{\mu}^{*}$, if $u_{0}(x) \leq U_{\mu_{0}}(x)$, $\lim _{t \rightarrow \infty} u(x, t)=0$ and $\lim _{t \rightarrow \infty} v(x, t)=\mu$ uniformly for $x \in \bar{\Omega}$.

(5) Suppose that $C_{\mu}$ is given by (4.42) and $U_{0}(x)$ is as defined in Proposition 4.10. Then for $u_{0}(x) \geq \sigma^{\prime} U_{0}(x)$, where $\sigma^{\prime}=\max \left\{\mu+c / m, C_{\mu+c / m} /\left\|U_{0}\right\|_{\infty}\right\}$, (4.47) holds.

Proof. The estimate of $v(x, t)$ in (4.46) is the same as before. For Part 1, let $u_{1}(x, t)$ be the solution of (3.29) with the same $u_{0}$ but with $\mu$ replaced by $\mu+c / m$. Then $u(x, t)>u_{1}(x, t)$ for $t>0$ from the comparison principle. Thus the blow-up of $u(x, t)$ follows from Theorem 4.9, When $\mu<-c / m, v(x, t) \rightarrow 0$ can be seen from (4.46). The proof of Part 2 is similar to that of Theorem 3.8. The proofs of Parts $3-5$ are simple applications of the comparison principle, (4.46), Theorem 4.9 and Proposition 4.10

4.5. Discussions. In this section, a strong prey growth rate $\lambda>\lambda_{1}^{D}\left(\Omega_{0}\right)$ is imposed. As a result, unbounded growth of the prey inside $\Omega_{0}$ becomes possible for any given predator growth rate $\mu$ :

(1) If $\mu \leq \mu_{*}^{0}-c / m$, then this happens for any given initial prey population $u_{0}$ (see Part 1 of Theorem 4.11).

(2) For $\mu>\mu_{*}^{0}-c / m$, if $u_{0}(x) \geq U_{\mu_{0}}(x)$ (Part 3 of Theorem 4.11) or if $u_{0}(x) \geq \sigma^{\prime} U_{0}(x)$ (Part 5 of Theorem 4.11), then $u(x, t)$ also blows up in $\Omega_{0}$.

The second case above means that no matter how large the predator growth rate is, the prey population can blow up for certain initial population distributions. Moreover, by our discussion on the free boundary problem at the end of subsection 4.3, and by Part 5 in Theorem 4.11 and Proposition 4.10, even if the introduction of a new prey is initially restricted to a very narrow region in the habitat (but perhaps with a high density), unbounded growth of this prey population is still possible if its growth rate $\lambda$ is high enough. This is of particular interest for understanding the biological invasion of a new species, since it shows that the presence of a very strong predator cannot stop the invasion if there exists an ideal environment $\Omega_{0}$ which sufficiently nutrients the growth of the new prey population. This contrasts with the studies in $\mathrm{OL}$, in which predation can slow, stall or reverse the invasion 
of the prey population; but they use rather different reaction functions from our model.

On the other hand, when $m$ is large, there exists an intermediate $\mu$ such that the prey population is persistent and stays bounded if its initial size $u_{0}$ is relatively small (Part 2 of Theorem 4.11). Thus, in view of case 2 above, for such a $\mu$, the system exhibits an Allee effect. Furthermore, if $\mu$ is large, the prey will die out if its initial population is small (Part 4 of Theorem 4.11). Thus, for large $\mu$, the prey population can either blow up (if $u_{0}$ is large) or die out (if $u_{0}$ is small).

Parts 3 and 4 of Theorem 4.11 also have other interesting biological explanations. These results show that for the initial population distribution $u_{0}=U_{\mu_{0}}$ (determined by Part 3 of Theorem 4.11), the prey population will become extinct when the predator is strong $\left(\mu>\widetilde{\mu}^{*}\right)$, but for the same initial distribution, the prey population can also blow up if the predator is weak $(\mu<\widehat{\mu}-c / m)$. Hence no initial distribution is guaranteed to predict extinction or blow-up of the prey population. We remark that this idea can also be applied to the weak prey growth case considered in Section 3, to show that the same initial distribution can lead to either extinction or persistence depending on the predator growth rate.

Finally we notice that in the weak prey growth case, the possibility of an Allee effect depends on the value of $m$, the handling time of the predator on each prey. When $m$ is large, two attracting regions exist for the system, but when $m$ is small, a unique attracting region will absorb all initial values. In sharp contrast, in the strong prey growth case, bistability is always possible no matter how small $m$ is. Hence in the latter case the Allee effect is mainly caused by the degeneracy of $a(x)$, not the rate of saturation of the predation. In Section 5, we shall show that for any $m$, strong but bounded growth is still possible if $a(x)$ is nearly degenerate in a subregion $\Omega_{0}$.

\section{Perturbed system}

In this section we consider a perturbation of (2.1) and (2.2):

$$
\begin{cases}u_{t}-\Delta u=\lambda u-[a(x)+\varepsilon] u^{2}-\frac{b u v}{1+m u}, & x \in \Omega, t>0, \\ v_{t}-\Delta v=\mu v-v^{2}+\frac{c u v}{1+m u}, & x \in \Omega, t>0, \\ \partial_{\nu} u=\partial_{\nu} v=0, & x \in \partial \Omega, t>0, \\ u(x, 0)=u_{0}(x) \geq 0, v(x, 0)=v_{0}(x) \geq 0, & x \in \Omega,\end{cases}
$$

and

$$
\begin{cases}-\Delta u=\lambda u-[a(x)+\varepsilon] u^{2}-\frac{b u v}{1+m u}, & x \in \Omega, \\ -\Delta v=\mu v-v^{2}+\frac{c u v}{1+m u}, & x \in \Omega, \\ \partial_{\nu} u=\partial_{\nu} v=0, & x \in \partial \Omega .\end{cases}
$$

We are interested in the spatial pattern of steady state solutions when $\varepsilon \rightarrow 0$ and related dynamics. Throughout the section, we assume the strong prey growth $\lambda>\lambda_{1}^{D}\left(\Omega_{0}\right)$.

5.1. Steady state solutions. First we use degree theory to study the steady state solutions of (5.2) for small $\varepsilon$. 
Proposition 5.1. Suppose that $\lambda>\lambda_{1}^{D}\left(\Omega_{0}\right)$ and that $b, c, m>0$ are fixed. Then for any large constant $M>0$, there exists $\varepsilon_{0}>0$ such that for $\varepsilon \in\left(0, \varepsilon_{0}\right)$, (5.2) has a positive solution $\left(u_{\mu, \varepsilon}^{2}, v_{\mu, \varepsilon}^{2}\right)$ when $\mu \in(\lambda / b, M]$. Moreover, for any sequence $\varepsilon_{n} \rightarrow 0$, subject to choosing a subsequence,

$$
u_{\mu, \varepsilon_{n}}^{2} \rightarrow u_{\mu}(x), \quad v_{\mu, \varepsilon_{n}}^{2} \rightarrow v_{\mu}(x), \quad \text { uniformly for } x \in \bar{\Omega},
$$

where $\left(u_{\mu}, v_{\mu}\right)$ is a positive solution of (2.2).

Proof. Recall the definitions $E=C(\bar{\Omega})$ and $P=\{u \in E: u(x) \geq 0, x \in \bar{\Omega}\}$. From Proposition 4.1, when $\mu \leq M$, there exists $C>0$ such that any nonnegative solution $(u, v)$ of (2.2) satisfies $\|u\|_{\infty}+\|v\|_{\infty}<C$. Let $T=\left\{(u, v) \in P^{2}: u \leq C, v \leq C\right\}$. We define $A_{\mu, \varepsilon}(u, v): T \rightarrow P^{2}$ by

$$
A_{\mu, \varepsilon}(u, v)=\left(\begin{array}{c}
(-\Delta+\alpha)^{-1}\left((\lambda+\alpha) u-(a(x)+\varepsilon) u^{2}-\frac{b u v}{1+m u}\right) \\
(-\Delta+\alpha)^{-1}\left((\mu+\alpha) v-v^{2}+\frac{c u v}{1+m u}\right)
\end{array}\right),
$$

where $\alpha>0$ is large enough such that $A_{\mu, \varepsilon}$ maps $T$ into $P^{2}$ for all $\mu \in[-c / m-1, M]$ and $\epsilon \in[0,1]$. It is well known that $A_{\mu, \varepsilon}$ is completely continuous and differentiable. For fixed $(\mu, \varepsilon)$, the fixed points of $A_{\mu, \varepsilon}(u, v)$ in $T$ are the nonnegative solutions of (5.2) (or (2.2) if $\varepsilon=0$ ).

By our choice of $T, A_{\mu, 0}(u, v) \neq(u, v)$ if $\mu \in[-c / m-1, M]$ and $(u, v) \in \partial T$ (the relative boundary of $T$ in $\left.P^{2}\right)$. By a standard compactness argument we can find some $\epsilon_{0}>0$ such that

$$
A_{\mu, \epsilon}(u, v) \neq(u, v) \text { for } \varepsilon \in\left(0, \varepsilon_{0}\right], \mu \in[-c / m-1, M],(u, v) \in \partial T .
$$

Therefore the fixed point index (relative to the cone $\left.P^{2}\right) \operatorname{ind}\left(A_{\mu, \varepsilon}, T\right)$ is well defined and is independent of $(\varepsilon, \mu)$ in the range given above.

Now we use the invariance of the fixed point index to show that for $\varepsilon \in\left(0, \varepsilon_{0}\right]$ and $\mu \in(\lambda / b, M]$, (5.2) has a positive solution. For $\mu<0$, (5.2) has a constant solution $(0,0)$, and for any $\mu>0,(5.2)$ has two constant solutions $(0,0)$ and $(0, \mu)$ in $T$. By the fixed point index calculation result of E. N. Dancer (see [Da1] and Da2]), the index of $(0,0)$ (as an isolated fixed point in $T$ ) is always 0 . The index of $(0, \mu)$ is 0 when $\mu<\lambda / b$, and it is 1 when $\mu>\lambda / b$. On the other hand, when $\mu<-c / m$, the only solution of (5.2) in $T$ is $(0,0)$ for any $\varepsilon \in\left(0, \varepsilon_{0}\right]$; thus $\operatorname{ind}\left(A_{\mu, \varepsilon}, T\right)=0$ when $\mu<-c / m$. From the invariance of the fixed point index with respect to $\mu$, for $\mu_{1}<-c / m$ and $\mu \in(\lambda / b, M]$,

$$
0=\operatorname{ind}\left(A_{\mu_{1}, \varepsilon}, T\right)=\operatorname{ind}\left(A_{\mu, \varepsilon}, T\right) .
$$

If at $\mu \in(\lambda / b, M]$, (5.2) has no solutions other than $(0,0)$ and $(0, \mu)$ in $T$, then

$$
\operatorname{deg}\left(A_{\mu, \varepsilon}, T\right)=\operatorname{ind}\left(A_{\mu, \varepsilon},(0,0)\right)+\operatorname{ind}\left(A_{\mu, \varepsilon},(0, \mu)\right)=0+1=1,
$$

which is a contradiction with (5.5). Therefore (5.2) has another solution $\left(u_{\mu, \varepsilon}^{2}, v_{\mu, \varepsilon}^{2}\right)$ in $T$, which is necessarily a positive solution. Finally since any positive solution satisfies $v>\mu$, and $(0, \mu)$ is an isolated solution of (5.2) and (2.2), when $\varepsilon_{n} \rightarrow 0$, by a standard compactness argument, subject to a subsequence, $\left\{\left(u_{\mu, \varepsilon_{n}}^{2}, v_{\mu, \varepsilon_{n}}^{2}\right)\right\}$ converges to a positive solution of (2.2).

Now we are ready for the main result on the steady state solutions: 
Theorem 5.2. Suppose that $\lambda>\lambda_{1}^{D}\left(\Omega_{0}\right)$ and that $b, c, m>0$ are fixed. Then for any large constant $M>0$ and small constant $\delta>0$, there exists $\varepsilon_{0}>0$ such that for any $\varepsilon \in\left(0, \varepsilon_{0}\right)$, (5.2) has a positive solution $\left(u_{\mu, \varepsilon}^{1}, v_{\mu, \varepsilon}^{1}\right)$ when $\mu \in\left[\mu_{*}+\delta, M\right]$, which satisfies

$$
\begin{aligned}
& \min \left\{\bar{U}_{\mu, \varepsilon}(x), \bar{U}_{0, \varepsilon}(x)\right\}>u_{\mu, \varepsilon}^{1}(x)>\bar{U}_{\mu+c / m, \varepsilon}(x), x \in \Omega, \\
& \mu+\frac{c}{m}>v_{\mu, \varepsilon}^{1}(x)>\max \{\mu, 0\}, x \in \Omega,
\end{aligned}
$$

where $\bar{U}_{\mu, \varepsilon}$ is the maximal solution of (4.22) and

$$
\mu_{*}=\lambda_{1}^{N}\left(-\Phi_{0}\right),\left.\Phi_{0}\right|_{\bar{\Omega}_{0}}=c / m,\left.\Phi_{0}\right|_{\bar{\Omega} \backslash \bar{\Omega}_{0}}=c \underline{U}^{\lambda} /\left(1+m \underline{U}^{\lambda}\right) .
$$

Moreover, (5.2) has another positive solution $\left(u_{\mu, \varepsilon}^{2}, v_{\mu, \varepsilon}^{2}\right)$ when $\mu \in(\lambda / b, M]$. Furthermore, for any sequence $\varepsilon_{n} \rightarrow 0$, subject to choosing a subsequence,

$$
\begin{aligned}
& u_{\mu, \varepsilon_{n}}^{1} \rightarrow \infty \quad \text { uniformly in } \overline{\Omega_{0}}, \quad v_{\mu, \varepsilon_{n}}^{1} \rightarrow V \quad \text { uniformly in } \bar{\Omega}, \\
& u_{\mu, \varepsilon_{n}}^{1} \rightarrow U \quad \text { uniformly on any compact subset of } \bar{\Omega} \backslash \overline{\Omega_{0}},
\end{aligned}
$$

where $(U, V)$ is a positive solution of the problem

$$
\left\{\begin{array}{l}
-\Delta U=\lambda U-a(x) U^{2}-\frac{b U V}{1+m U} \text { in } \Omega \backslash \bar{\Omega}_{0} ;\left.U\right|_{\partial \Omega_{0}}=\infty,\left.\partial_{\nu} U\right|_{\partial \Omega}=0 \\
-\Delta V=(\mu+\Phi(x)) V-V^{2} \text { in } \Omega,\left.\quad \partial_{\nu} V\right|_{\partial \Omega}=0
\end{array}\right.
$$

with $\Phi(x)=c / m$ in $\Omega_{0}$ and $\Phi(x)=C U /(1+m U)$ in $\Omega \backslash \Omega_{0}$, and

$$
u_{\mu, \varepsilon_{n}}^{2} \rightarrow u_{\mu}, \quad v_{\mu, \varepsilon_{n}}^{2} \rightarrow v_{\mu} \quad \text { uniformly in } \bar{\Omega}
$$

where $\left(u_{\mu}, v_{\mu}\right)$ is a positive solution of (2.2).

Proof. Recall that $\hat{\mu}_{\varepsilon}^{*}=\sup \{\mu>0:$ (4.22) has a positive solution $\}$, and define $\widetilde{\mu}_{\varepsilon}^{*}=\sup \{\mu>0:(5.2)$ has a positive solution $\}$. Then from the proof of Theorem 3.5. Part 1 , we have $\widetilde{\mu}_{\varepsilon}^{*} \leq \hat{\mu}_{\varepsilon}^{*}$. Now we can follow the same proof as that of Theorem 3.5 to show that when $\mu \in\left(\lambda_{1}^{N}\left(-c p\left(u_{\lambda, \varepsilon}\right)\right), \hat{\mu}_{\varepsilon}^{*}-c / m\right]$, (5.2) has a positive solution $\left(u_{\mu, \varepsilon}^{1}, v_{\mu, \varepsilon}^{1}\right)$ satisfying (5.7). We notice that when $\varepsilon \rightarrow 0, \lambda_{1}^{N}\left(-c p\left(u_{\lambda, \varepsilon}\right)\right) \rightarrow \mu_{*}$ as $\varepsilon \rightarrow 0$ since $u_{\lambda, \varepsilon} \rightarrow \infty$ uniformly for $x \in \overline{\Omega_{0}}$, and $u_{\lambda, \varepsilon} \rightarrow \underline{U}^{\lambda}$ locally uniformly on $\bar{\Omega} \backslash \bar{\Omega}_{0}$; thus the lower bound of the $\mu$-range for the existence of $\left(u_{\mu, \varepsilon}^{1}, v_{\mu, \varepsilon}^{1}\right)$ can be smaller than any $\mu_{*}+\delta$ if $\varepsilon$ is small enough. By Proposition [5.1, the upper bound $\widetilde{\mu}_{\varepsilon}^{*}$ can be chosen as large as possible if $\varepsilon$ is small enough. Thus we may assume $\widetilde{\mu}_{\varepsilon}^{*}>M+c / m$, and the upper bound of the $\mu$-range for the existence of $\left(u_{\mu, \varepsilon}^{1}, v_{\mu, \varepsilon}^{1}\right)$ is then at least $M$. The estimate of $u_{\mu, \varepsilon}^{1}$ in (5.8) for $x \in \bar{\Omega}_{0}$ follows from Proposition 4.7. and the other estimate for $u_{\mu, \varepsilon}^{1}$ and $v_{\mu, \varepsilon}^{1}$ can be shown in the following way.

Denote $\Phi_{n}=c u_{\mu, \varepsilon_{n}}^{1} /\left(1+m u_{\mu, \varepsilon_{n}}^{1}\right)$. Then $\Phi_{n}$ is bounded in $L^{\infty}(\Omega)$, and by passing to a subsequence, we may assume that $\Phi_{n} \rightarrow \Phi$ weakly in $L^{2}(\Omega)$. Now from the equation for $v_{\mu, \epsilon_{n}}^{1}$ we see that it is a bounded sequence in $W^{2, p}(\Omega)$ for any $p>1$ and hence, by passing to a subsequence, $v_{\mu, \epsilon_{n}}^{1} \rightarrow V$ in $C^{1}(\bar{\Omega})$, where $V$ is a positive solution of

$$
-\Delta V=(\mu+\Phi) v-v^{2} \text { in } \Omega,\left.\partial_{\nu} V\right|_{\partial \Omega}=0 .
$$

Coming back to the equation for $u_{\mu, \varepsilon_{n}}^{1}$ over $\Omega \backslash \bar{\Omega}_{0}$, since $u_{\mu, \varepsilon_{n}}^{1} \leq u_{\lambda, \varepsilon}$, by a standard compactness argument, we can show that, subject to passing to a subsequence, 
$u_{\mu, \varepsilon}^{1} \rightarrow U$ uniformly in any compact subset of $\bar{\Omega} \backslash \overline{\Omega_{0}}$, where $U$ is a positive solution of

$$
-\Delta U=\lambda U-a(x) U^{2}-\frac{b U V}{1+m U} \text { in } \Omega \backslash \bar{\Omega}_{0} ;\left.U\right|_{\partial \Omega_{0}}=\infty,\left.\partial_{\nu} U\right|_{\partial \Omega}=0 .
$$

It follows that $\Phi(x)=c U /(1+m U)$ in $\Omega \backslash \bar{\Omega}_{0}$. Since $u_{\mu, \varepsilon_{n}} \rightarrow \infty$ in $\Omega_{0}$, we must have $\Phi(x)=c / m$ in $\Omega_{0}$.

Finally the existence and estimate of $\left(u_{\mu, \varepsilon}^{2}, v_{\mu, \varepsilon}^{2}\right)$ follows from Proposition 5.1 .

The corresponding dynamical behavior can be formulated following the pattern in Theorem 3.8, where Theorem 3.6] was used to understand (3.29); here we make use of Theorem 4.8 instead. We leave the details to the interested reader. We also comment that the existence of three positive solutions of (5.2) for certain $\mu$ can be proved if $m<m_{0}$, in a similar fashion as in Theorem 3.5 .

5.2. Discussion. Theorem 5.2 implies that the range of the predator growth rate $\mu$ for which the system (5.1) has a bistable dynamical structure can be very large if the parameter $\varepsilon$ is small enough. At the same time, the coexistence steady state $\left(u_{\mu, \varepsilon}^{1}, v_{\mu, \varepsilon}^{1}\right)$ shows a clear spatial pattern when $\varepsilon \rightarrow 0$, where the prey population reaches a carrying capacity in the order of $O\left(\varepsilon^{-1}\right)$ uniformly on $\Omega_{0}$, the favorable subregion for the prey. We remark that similar results can also be proved if $\Omega_{0}$ is replaced by finitely many disjoint subregions $\Omega_{i}(i=1,2, \cdots, k)$, following the methods in DHs, DLi. On the other hand, our studies show that if we design a certain subregion $\Omega_{0}$ so that the crowding effect of the prey is small there, then abundance of the prey population can be achieved, and this is of interest for commercially valuable prey species. Designing the best subregion $\Omega_{0}$ is also of recent interest; see $\mathrm{KuS}$.

Finally we prove the statement after Proposition 3.4 about the possibility of a reversed $S$-shaped bifurcation diagram and existence of three positive solutions. Indeed from the construction there, when $\varepsilon=0$, the global continuum $\widetilde{\Sigma}_{0}$ of positive solutions to (3.12) is unbounded from Theorem 4.2 since $\lambda>\lambda_{1}^{D}\left(\Omega_{2}\right)$ and $a_{0}(x)=0$ on $\Omega_{2}$. Next we use the proof of Proposition 5.1 to show that when $\varepsilon>0$ is small, the global continuum $\widetilde{\Sigma}_{\varepsilon}$ of positive solutions to (3.12) can be extended to any $M>0$ as long as $\varepsilon$ is small. In particular when $\varepsilon>0$ is small, we have $\hat{\mu}^{*}(m)>\lambda / b>\hat{\mu}_{*}(m)$, and this can be achieved for any $m>0$ and $\lambda>\lambda_{1}^{D}(\Omega)$. This construction shows that when $a(x)$ is defined roughly by

$$
a(x)= \begin{cases}0, & x \in \Omega_{0}, \\ \text { small, } & x \in \Omega_{2} \backslash \overline{\Omega_{0}}, \\ \text { large, } & x \in \Omega \backslash \overline{\Omega_{2}},\end{cases}
$$

then a reversed $S$-shaped bifurcation diagram is possible, and the complexity of the dynamics is caused not only by the degeneracy on $\Omega_{0}$, but also by the near degeneracy on $\Omega_{2} \backslash \overline{\Omega_{0}}$.

\section{ACKNowledgement}

The authors would like to thank the referee for a careful reading of the manuscript and insightful suggestions which helped us to improve the biological interpretations of our results. J. Shi is also thankful for the support and hospitality of the University of New England while this work was being done. 


\section{REFERENCES}

[BL] Berestycki, H.; Lions, P.-L., Some applications of the method of super and subsolutions. Lecture Notes in Math., 782, Springer, Berlin, 1980, pp. 16-41. MR.572249 (82c:35021)

[BNV] Berestycki, H.; Nirenberg, L.; Varadhan, S.R.S., The principle eigenvalue and maximum principle for second-order elliptic operators in general domains. Comm. Pure Appl. Math. 47 (1994), no. 1, 47-92. MR1258192 (95h:35053)

[BB] Blat, J.; Brown, K.J., Global bifurcation of positive solutions in some systems of elliptic equations. SIAM J. Math. Anal., 17 (1986), 1339-1353. MR860917 (87k:35077)

[BD] Brown, K. J.; Du, Yihong, Bifurcation and monotonicity in competition reaction-diffusion systems. Nonlinear Anal., 23 (1994), 1-13. MR1288495 (95h:35112)

[CC1] Cantrell, Robert Stephen; Cosner, Chris, Diffusive logistic equations with indefinite weights: Population models in disrupted environments. Proc. Roy. Soc. Edinburgh Sect. A 112 (1989), no. 3-4, 293-318. MR.1014659 (91b:92015)

[CC2] Cantrell, Robert Stephen; Cosner, Chris, Spatial ecology via reaction-diffusion equations. Wiley Series in Mathematical and Computational Biology, John Wiley \& Sons Ltd., 2003. MR 2191264

[CEL] Casal, A.; Eilbeck, J. C.; López-Gómez, J. Existence and uniqueness of coexistence states for a predator-prey model with diffusion. Differential Integral Equations 7 (1994), no. 2, 411-439. MR.1255897 (95f:92009)

[CP] Clément, Ph.; Peletier, L. A., An anti-maximum principle for second-order elliptic operators. J. Differential Equations, 34, (1979), no. 2, 218-229. MR.550042 (83c:35034)

[Co] Conway, E. D., Diffusion and predator-prey interaction: pattern in closed systems. Partial differential equations and dynamical systems, 85-133, Res. Notes in Math., 101, Pitman, Boston, Mass.-London, (1984). MR759745 (86e:92035)

[CR1] Crandall, Michael G.; Rabinowitz, Paul H., Bifurcation from simple eigenvalues. J. Functional Analysis, 8 (1971), 321-340. MR0288640 (44:5836)

[CR2] Crandall, Michael G.; Rabinowitz, Paul H., Bifurcation, perturbation of simple eigenvalues and linearized stability. Arch. Rational Mech. Anal. 52 (1973), 161-180. MR0341212 (49:5962)

[Da1] Dancer, E. N., On the indices of fixed points of mappings in cones and applications, $J$. Math. Anal. Appl., 91 (1983), 131-151. MR688538 (84d:58020)

[Da2] Dancer, E. N., On positive solutions of some pairs of differential equations. Trans. Amer. Math. Soc. 284 (1984), no. 2, 729-743. MR.743741 (85i:35056)

[DD] Dancer, E. N.; Du, Yihong, Effects of certain degeneracies in the predator-prey model. SIAM J. Math. Anal. 34 (2002), no. 2, 292-314. MR1951776 (2004a:35064)

[dMR] de Mottoni, P.; Rothe, F., Convergence to homogeneous equilibrium state for generalized Volterra-Lotka systems with diffusion. SIAM J. Appl. Math. 37(1979), 648-663. MR549146 (81b:92008)

[D1] Du, Yihong, Effects of a degeneracy in the competition model. I. Classical and generalized steady-state solutions. J. Differential Equations 181 (2002), no. 1, 92-132. MR1900462 (2003d:35125)

[D2] Du, Yihong, Effects of a degeneracy in the competition model. II. Perturbation and dynamical behaviour. J. Differential Equations 181 (2002), no. 1, 133-164. MR1900463 (2003d:35124)

[D3] Du, Yihong, Realization of prescribed patterns in the competition model. J. Differential Equations 193 (2003), no. 1, 147-179. MR1994062 (2004j:35095)

[D4] Du, Yihong, Spatial patterns for population models in a heterogeneous environment. Taiwanese J. Math. 8 (2004), no. 2, 155-182. MR2061685(2005e:92035)

[D5] Du, Yihong, Bifurcation and related topics in elliptic problems. Stationary partial differential equations. Vol. II, 127-209, Handb. Differ. Equ., Elsevier/North-Holland, Amsterdam, 2005. MR2181483 (2006k:35113)

[D6] Du, Yihong, Asymptotic behavior and uniqueness results for boundary blow-up solutions. Diff. Integral Eqns., 17(2004), 819-834. MR2074688 (2005f:35096)

[DHs] Du, Yihong; Hsu, Sze-Bi, A diffusive predator-prey model in heterogeneous environment. J. Differential Equations 203 (2004), no. 2, 331-364. MR2073690(2005e:35051)

[DHu] Du, Yihong; Huang, Qingguang, Blow-up solutions for a class of semilinear elliptic and parabolic equations. SIAM J. Math. Anal. 31 (1999), no. 1, 1-18. MR1720128(2000g:35059) 
[DLi] Du, Yihong; Li, Shujie, Positive solutions with prescribed patterns in some simple semilinear equations. Differential Integral Equations 15 (2002), no. 7, 805-822. MR1895567 (2003f:35105)

[DL1] Du, Yihong; Lou, Yuan, Some uniqueness and exact multiplicity results for a predator-prey model. Trans. Amer. Math. Soc. 349 (1997), no. 6, 2443-2475. MR1401768 (98e:35164)

[DL2] Du, Yihong; Lou, Yuan, S-shaped global bifurcation curve and Hopf bifurcation of positive solutions to a predator-prey model. J. Differential Equations 144 (1998), no. 2, 390-440. MR.1616901 (99e:35046)

[DL3] Du, Yihong; Lou, Yuan, Qualitative behaviour of positive solutions of a predator-prey model: Effects of saturation. Proc. Roy. Soc. Edinburgh Sect. A 131 (2001), no. 2, 321349. MR 1830414 (2002e:35125)

[DM] Du, Yihong; Ma, Li, Logistic type equations on $\mathbf{R}^{N}$ by a squeezing method involving boundary blow-up solutions. J. London Math. Soc. (2) 64 (2001), no. 1, 107-124. MR 1840774 (2002d:35089)

[DS1] Du, Yihong; Shi, Junping, Spatially Heterogeneous Predator-Prey Model with Protect Zone for Prey. Jour. Diff. Equa., 229 (2006), no. 1, 63-91. MR.2265618

[DS2] Du, Yihong; Shi, Junping, Some Recent Results on Diffusive Predator-prey Models in Spatially Heterogeneous Environment. Nonlinear dynamics and evolution equations, Fields Institute Communications, 48, American Mathematical Society (2006), pp. 95-135. MR 2223350

[H] Holling, C. S., Some characteristics of simple types of predation and parasitism. Canadian Entomologist 91 (1959), 385-398.

[HLR] Huang, Jianhua; Lu, Gang; Ruan, Shigui, Existence of traveling wave solutions in a diffusive predator-prey model. J. Math. Biol. 46 (2003), no. 2, 132-152. MR1963069 (2004b:35183)

[KS] Korman, Philip; Shi, Junping, New exact multiplicity results with an application to a population model. Proc. Royal Soc. Edin. A, 131 (2001), No. 5, 1167-1182. MR.1862448 (2002h:35092)

[KuS] Kurata, Kazuhiro; Shi, Junping, Optimal spatial harvesting strategy and symmetrybreaking. Submitted, (2005).

[MP] Medvinsky, Alexander B.; Petrovskii, Sergei V.; Tikhonova, Irene A.; Malchow, Horst; Li, Bai-Lian, Spatiotemporal complexity of plankton and fish dynamics. SIAM Rev. 44 (2002), no. 3, 311-370. MR1951363 (2003m:92083)

[OSS] Oruganti, Shobha, Shi, Junping; Shivaji, Ratnasingham, Diffusive logistic equation with constant yield harvesting, I: Steady States. Trans. Amer. Math. Soc., 354, (2002), no. 9, 3601-3619,. MR1911513 (2003g:35080)

[O] Ouyang, Tiancheng, On the positive solutions of semilinear equations $\Delta u+\lambda u-h u^{p}=0$ on the compact manifolds. Trans. Amer. Math. Soc. 331 (1992), no. 2, 503-527. MR1055810 (92h:35012)

[OS1] Ouyang, Tiancheng; Shi, Junping, Exact multiplicity of positive solutions for a class of semilinear problems. Jour. Diff. Equa. 146 (1998), no. 1, 121-156. MR1625731 (99f:35061)

[OS2] Ouyang, Tiancheng; Shi, Junping, Exact multiplicity of positive solutions for a class of semilinear problem: II. Jour. Diff. Equa. 158, (1999), no. 1, 94-151. MR1721723 (2001b:35117)

[OL] Owen, M.R.; Lewis, M.A., How can predation slow, stall or reverse a prey invasion? Bull. Math. Biol. 63, (2001), 655-684.

[SJ] Segel, L.A.; Jackson, J.L., Dissipative structure: An explaination and an ecological example. J. Theor. Biol., 37, (1975), 545-559.

[SL] Segel, Lee A.; Levin, Simon A. Application of nonlinear stability theory to the study of the effects of diffusion on predator-prey interactions. AIP Conf. Proc., 27, Amer. Inst. Phys., New York, (1976), 123-156.. MR0496857 (58:15316)

[S1] Shi, Junping, Persistence and bifurcation of degenerate solutions. Jour. Funct. Anal. 169, (1999), No. 2, 494-531. MR1730558 (2001h:47115)

[S2] Shi, Junping, A radially symmetric anti-maximum principle and applications to fishery management models. Electronic Journal of Differential Equations. 2004, No. 27, 1-13, (2004). MR2036211 (2004k:34031) 
[SS1] Shi, Junping; Shivaji, Ratnasingham, Global bifurcations of concave semipositone problems. Advances in Evolution Equations: Proceedings in honor of J. A. Goldstein's 60th birthday, Edited by G.R. Goldstein, R. Nagel, and S. Romanelli, Marcel Dekker, Inc., New York, Basel, 385-398, (2003). MR2073759 (2005g:35113)

[SS2] Shi, Junping; Shivaji, Ratnasingham, Persistence in reaction diffusion models with weak Allee effect. Jour. Math. Biol. 52 (2006), 807-829. MR.2235529

[SY] Shi, Junping; Yao, Miaoxin, On a singular nonlinear semilinear elliptic problem. Proc. Royal Soc. Edin. A, 128, (1998), no. 6, 1389-1401. MR1663988 (99j:35070)

[Si] Simon, Leon, Asymptotics for a class of nonlinear evolution equations, with applications to geometric problems. Ann. of Math. 118 (1983), no. 3, 525-571. MR727703(85b:58121)

School of Mathematics, Statistics and Computer Sciences, University of New England, Armidale, NSW2351, Australia - and - Department of Mathematics, Qufu Normal University, Qufu, Shandong 273165, People's Republic of China

E-mail address: ydu@turing.une.edu.au

Department of Mathematics, College of William and Mary, Williamsburg, Virginia 23187 - And - School of Mathematics, Harbin Normal University, Harbin, Heilongjiang 150025, People's Republic of China

E-mail address: shij@math.wm.edu 